\title{
Initial Feasibility Study of a Dedicated Synchrotron Radiation Light Source for Ultrafast X-Ray Science
}

\author{
J. N. Corlett, S. DeSantis, N. Hartman, P. Heimann, R. LaFever, D. Li, H. Padmore, R. Rimmer, \\ K. Robinson, R. Schoenlein, J. Tanabe, S. Wang and A. Zholents, \\ Ernest Orlando Lawrence Berkeley National Laboratory \\ 1 Cyclotron Road \\ Berkeley, California 94720, USA \\ and \\ D. Kairan, \\ Budker Institute for Nuclear Physics \\ 630090 Novosibirsk, Russia
}

This work was supported by the U.S.Department of Energy under Contract No. DE-AC03-76SF00098.

\begin{abstract}
We present an initial feasibility summary of a femtosecond synchrotron radiation x-ray source based on a flat-beam rf gun and a recirculating superconducting linac that provides beam to an array of undulators and bend magnets. Optical pulse durations of $<100 \mathrm{fs}$ are obtained by a combination of electron pulse compression, transverse temporal correlation of the electrons, and $\mathrm{x}$-ray pulse compression. After an introduction and initial scientific motivation, we cover the following aspects of the design: layout and lattice, ultra-fast $\mathrm{x}$-ray pulse production, flat electron-beam production, the rf gun, rf systems, cryogenic systems, collective effects, photon production, and synchronization of x-ray and laser pulses. We conclude with a summary of issues and areas of development that remain to be addressed.
\end{abstract}

\section{Introduction}

The advent of femtosecond lasers has revolutionized many areas of science from solid state physics to biology, and this has been recognized with the award of the 1999 Nobel prize in Chemistry to Ahmed Zewail for his pioneering work in application of these techniques to the study of ultra-fast chemical reactions. While optical lasers have offered unique insights into ultra-fast dynamics, the key information often needed, the motion of nuclei, is inaccessible unless detailed information is available through calculation of the excited state energy surface. This is a severe limitation in many cases, and has led to the development of ultra-fast electron diffraction systems to directly monitor atomic motion. While it has proven possible to generate picosecond electron pulses with typically tens of keV electron energy, the system resolution is typically several picoseconds, as a result of the fundamental velocity mismatch of the pump laser pulse and the probe electron pulse. This mismatch can only be reduced by an increase in the electron energy, but this correspondingly reduces the interaction cross section and makes the already long acquisition time for typical gas phase diffraction prohibitively long. The electron pulse duration itself is also far longer than many ultra-fast reactions. A breakthrough in the study of ultra-fast dynamics would be made if we can directly monitor the position of nuclei with $<100 \mathrm{fs}$ time resolution. For systems with no long range order we can use synchrotron radiation based Extended X-ray Absorption Fine Structure 
(EXAFS) spectroscopy. With this we can directly measure the distance to nearest neighbors from a particular emitting atom by monitoring the oscillations in $\mathrm{x}$-ray absorption occurring over typically hundreds of $\mathrm{eV}$ above the central atomic absorption edge. These oscillations are due to the reflection of the electron wave back to the central emitting atom, and so encode interatomic distances directly. Where long range order exists we can resort to $\mathrm{x}$-ray diffraction methodologies. The problem is to combine these techniques with an ultra-fast and intense $x$-ray pulse in the $<100 \mathrm{fs}$ regime.

It is this new research frontier of ultra-fast x-ray science that drives the development of a novel source for generating femtosecond $\mathrm{x}$-ray pulses. Recently, a number of important advances have been made in the generation of ultrashort $\mathrm{x}$-ray pulses using a variety of techniques, and while initial time-resolved $\mathrm{x}$ ray experiments have been performed using such sources, they suffer from significant limitations in one or more critical parameters including: (a) x-ray brightness and/or flux, (b) x-ray photon energy, tunability, and spectral range, and (c) x-ray pulse duration and control. Ultimately, the full scientific development of the emerging field of ultrafast $x$-ray science will require a dedicated facility supporting multiple beamlines for generating $\mathrm{x}$-rays with the highest possible $\mathrm{x}$-ray flux and brightness in the $0.3-10 \mathrm{keV}$ range, and with a pulse duration of less than $100 \mathrm{fs}$.

A source capable of giving the performance required, not just to observe ultra-fast dynamics in ordered materials but also to investigate liquids and disordered materials, is described here, and would represent a major step forward in ultra-fast dynamics research. The proposed source has the short pulse length necessary ( $50 \mathrm{fs}$ FHWM) to study very fast dynamics, the very high average flux to study weak scattering systems, tuneability in photon energy from approximately $1-10 \mathrm{keV}$, and a repetition rate matched to the requirements of structural dynamics experiments.

In this report we consider a facility which can be built on a basis of existing technologies and which has a potential to produce femtosecond x-ray pulses with high flux and brightness. The facility uses a recirculating linac for acceleration (and deceleration) of electrons produced by a flat-beam photocathode rf gun, at a bunch repetition rate up to approximately $50 \mathrm{kHz}$. The repetition rate is determined by either the time required for the experimental sample to return to its ground state (often limited by acoustic propagation velocities), the time to translate the sample to a new area after each shot, or the repetition rate of the possible attendant pump laser systems. The system design produces a small vertical emittance, which is key to producing the $\sim 50 \mathrm{fs}$-ray pulses. After acceleration through a superconducting recirculating accelerator with achromatic and isochronous arcs, the electron pulses are compressed in the final arc to $\sim 1$ ps duration. A specialized technique is then employed to produce the ultra-short $\mathrm{x}$-ray pulses by first imprinting on the electron bunches a transverse momentum that is correlated to longitudinal position within the bunch. This action causes a rotation of the individual bunches, and produces a photon pulse with a time-correlated transverse distribution when passing through an undulator or bend magnet. The correlated $\mathrm{x}$-ray pulse from such a beam is then compressed by use of crystal optics to achieve the ultimate photon pulse length. In order to obtain sub-100 fs x-ray pulses we thus use two stages of pulse compression compression of the electron pulse; then a direct compression of the x-ray pulse [ZHO99].

We describe the machine layout and lattice in Section 2, and techniques involved in producing photon pulses in the femtosecond regime are described in Section 3. The means by which a flat electron beam distribution is produced is described in Section 4. The photocathode RF gun is described in Section 5. We discuss the accelerating if systems with superconducting cavities and rf system power requirements in Section 6. This leads to a discussion of the cryogenic systems necessary and their attendant electrical 
power requirements (Section 7). We then present initial discussions of electron-beam collective effects (Section 8 ). The electron beam prepared to a $\sim 1$ ps pulse is transported into a photon beam production area where photons are produced in either undulator or bend magnets (Section 9). Synchronization of x-ray and laser pulses is discussed in section 10, and the concluding section (Section 11) discusses outstanding issues and planned future development.

\section{Lattice and Layout}

A layout of the present machine concept is shown in Figure 1. It consists of an rf photo-injector, a linear pre-accelerator, a main linear accelerator, magnetic arcs and straight sections, and a photon beam production section. Electron pulses of approximately $10 \mathrm{ps}$ duration are produced in a high-brightness rf photocathode gun and accelerated to $10 \mathrm{MeV}$. A magnetic field in the cathode region and a skewquadrupole channel are used to generate a "flat" beam with $\mathrm{x} / \mathrm{y}$ emittance ratio $\geq 50$ [BRI99, BRI01]. The electrons are further accelerated in a superconducting linac pre-accelerator to $120 \mathrm{MeV}$, and then injected into a recirculating linear accelerator. Passing the arc connecting the pre-accelerator and the recirculating linac the electron bunches are compressed to approximately 5 ps full length. In the recirculating linac the final energy of $\sim 2.5 \mathrm{GeV}$ is achieved after four passes through the $600 \mathrm{MeV}$ superconducting linac. After the fourth pass through the main linac, a final arc compresses the bunches to an approximately 1 ps length, and the bunches receive a time-correlated head-tail vertical kick in a dipole-mode rf cavity to obtain timecorrelated angular and transverse displacements. The electrons are then transported into the photon production section where they radiate $\mathrm{x}$-rays in undulators and dipole magnets. There are seven two-meter long straight sections for undulators in the production section as well as six $2 \mathrm{~T}$ field dipole magnets located between undulators each with a $60 \mathrm{mrad}$ arc angle. Each bend magnet can provide several photon beamlines. After passing through the photon production section, the electrons receive a compensating time-correlated head-tail vertical kick, are stretched back to approximately $5 \mathrm{ps}$. They are transported back to and decelerated in the recirculating accelerator structure and the $110 \mathrm{MeV}$ pre-accelerator to $\leq 10 \mathrm{MeV}$ and turned into the beam dump. This deceleration reduces the design challenges and radiation hazard of the beam dump. Though the electron beam does return almost all of its energy to the linac rf fields, the circulating beam power is so low that it does not contribute significantly to the overall system efficiency.

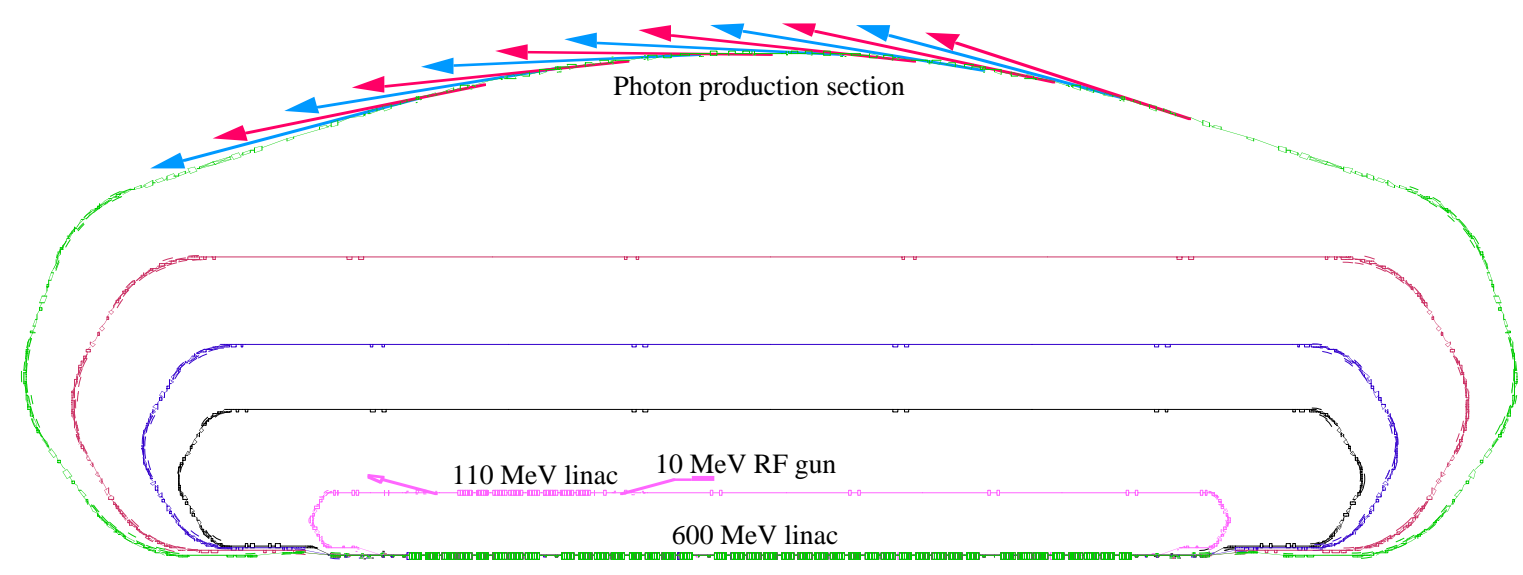

Figure 1. Recirculating linac for fs x-ray production 
Five arcs transport the electron beam with approximate energies of $120 \mathrm{MeV}(\operatorname{arc} 0), 720 \mathrm{MeV}$ (arc 1), $1.3 \mathrm{GeV}(\operatorname{arc} 2), 1.9 \mathrm{GeV}$ ( $\operatorname{arc} 3$ ), and $2.5 \mathrm{GeV}$ (arc 4). They are designed as achromats with almost identical lattices. All arcs are comprised of three $120^{\circ}$ betatron phase advance cells consisting of a set of bending magnets, three quadrupoles and three sextupoles. The bending magnets, quadrupoles and sextupoles of different arcs are mechanically identical except for the bending magnets in arc 0 . This lattice approach provides flexibility in tunability of the betatron phase advance, achromaticity including second order effects, and time-of-flight characteristics. The third cell of each arc leading to the straight section is adjusted to match the optical functions between the arc and it's corresponding straight section. At present, we have designed arcs 1 through 3 to be isochronous. They are, however, easily adapted should additional electron bunch length adjustment be required at the various stages of acceleration and deceleration. The number of linac passes and arcs allows us sufficient flexibility to optimally control the bunch length with consideration for collective effects such as beam break-up and coherent synchrotron radiation, as discussed in Section 8. We also retain the flexibility to manipulate the longitudinal charge density of the bunch, by control of the rf photocathode and incident laser parameters.

The separation of the different energy beams into their respective arcs is accomplished by a "beam spreader" lattice section, shown in Figure 2. The initial energy separation is accomplished by the dipole magnet B0. Magnet B1 acts only on the $720 \mathrm{MeV}$ beam (arc 1). The higher energy orbits pass through magnet B2. Magnets B1 and B2 have the opposite polarity from magnet B0 in order to limit the dispersion function excited in B0. The orbit separation between the $720 \mathrm{MeV}$ beam (arc 1) and $1.3 \mathrm{GeV}$ beam (arc 2) after magnets B1 and B2 is approximately $17 \mathrm{~cm}$. The orbit separation of the $1.3 \mathrm{GeV}(\operatorname{arc} 2)$ and $1.9 \mathrm{GeV}$ (arc 3) beams is approximately $18 \mathrm{~cm}$. This provides sufficient room for downstream quadrupoles by alternating their placement between the different arcs. The beam spreader quadrupoles differ from the regular arc quadrupole, Figure 3 shows a typical cross-section of a beam-spreader quadrupole (a) and a septum dipole (b). The small horizontal quadrupole dimension is obtained by using coils in the horizontal plane. Beam spreader quadrupoles are used to match the beta-functions at the exit of the linac to the arc lattice. Orbits of the $1.9 \mathrm{GeV}$ ( $\operatorname{arc} 3$ ) and $2.5 \mathrm{GeV}$ ( $\operatorname{arc} 4$ ) beams are separated by only $9.7 \mathrm{~cm}$ after the magnet B2, so dipole magnet B3 with the same field polarity as B2 is used to increase this separation. B3 is a septum dipole magnet with a high current density sheet on one side, that acts only on the $2.5 \mathrm{GeV}$ beam. A cross-section of this magnet is shown in Figure 3b. The magnet B4 completes the separation of the $2.5 \mathrm{GeV}$ beam, and together with magnets B0, B2, and B3 forms a chicane with no net dispersion or orbit displacement with respect to the linac exit. Four pairs of sextupoles are used in the beam spreader to control chromaticty. Apart from specialized magnets in the beam spreader section, the magnets are of conventional electromagnet design similar to those of existing synchrotron radiation sources. Magnet power requirements are approximately $2 \mathrm{MW}$.

Figure 4 shows lattice functions for the $1.9 \mathrm{GeV}$ beam beginning from the center of the $600 \mathrm{MeV}$ linac (a) and through the end of arc 3 (b). Lattice functions for other arcs show similar behavior. As can be seen in Figure 4, there are no focussing elements within the linac. The beam is constrained by quadrupoles and focussed to a relatively small size in the arc sections. The dispersion, generated in the beam spreader, changes polarity twice within the arc, and is brought to zero at the end of the arc. Adjustment of the amplitude of the negative dispersion with arc quadrupoles provides a simple way to control time-of-flight characteristics of each pass. 


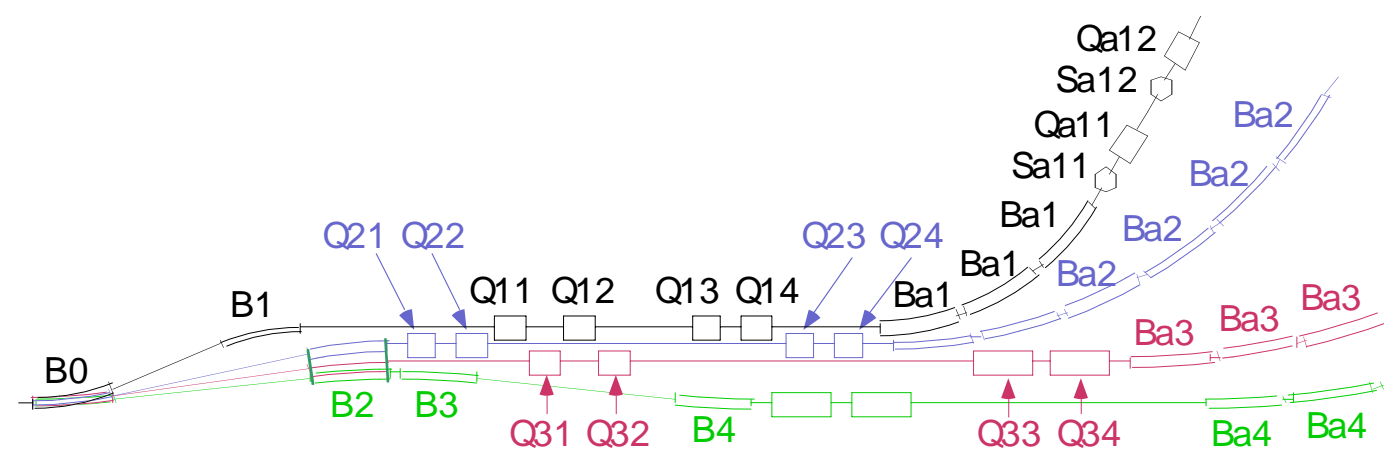

Figure 2. The beam spreader section that separates the beam at various energies into their respective transport paths.

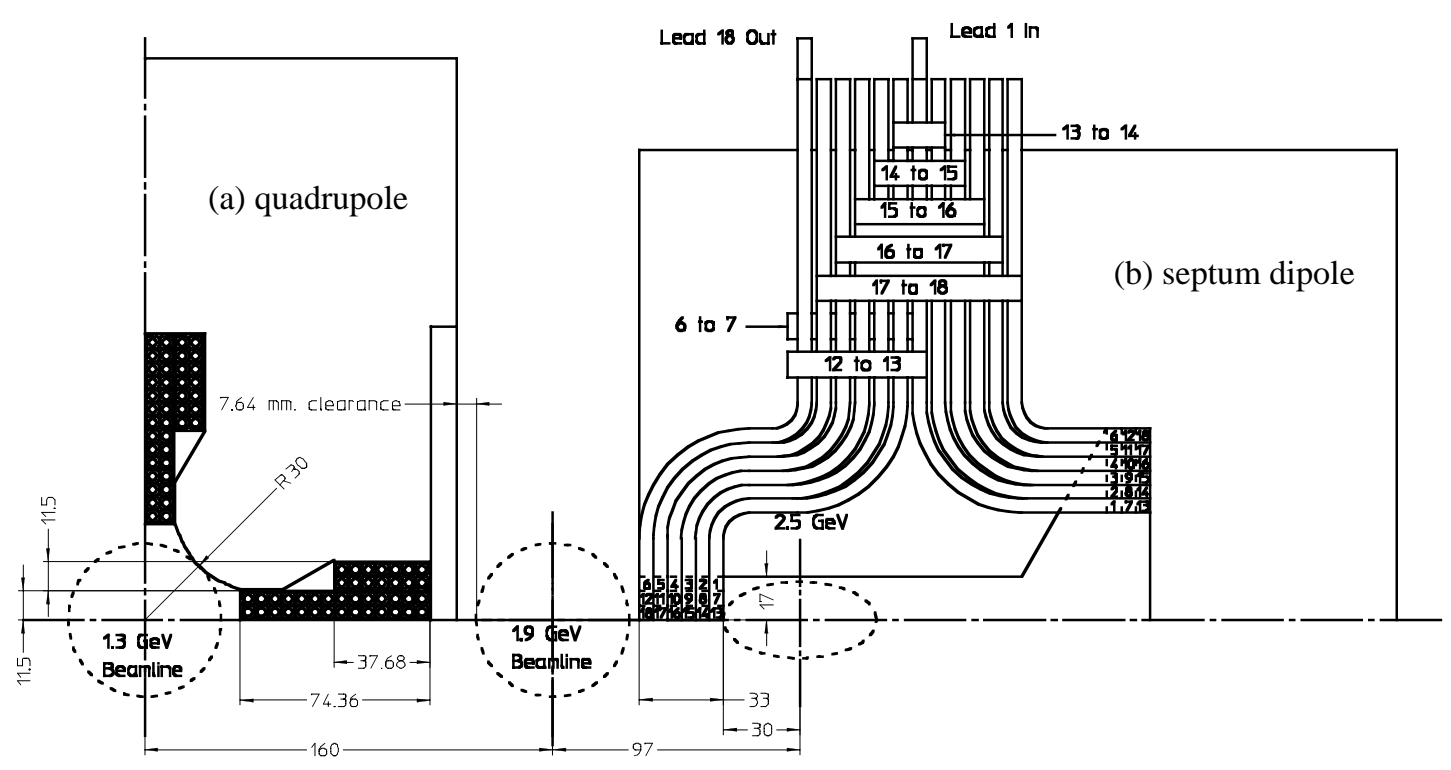

Figure 3. The beam spreader (a) quadrupole and (b) septum dipole magnets. The sectional drawing shows the physical separation of the three different energy beamlines with respect to the magnets. 


\section{(a)}

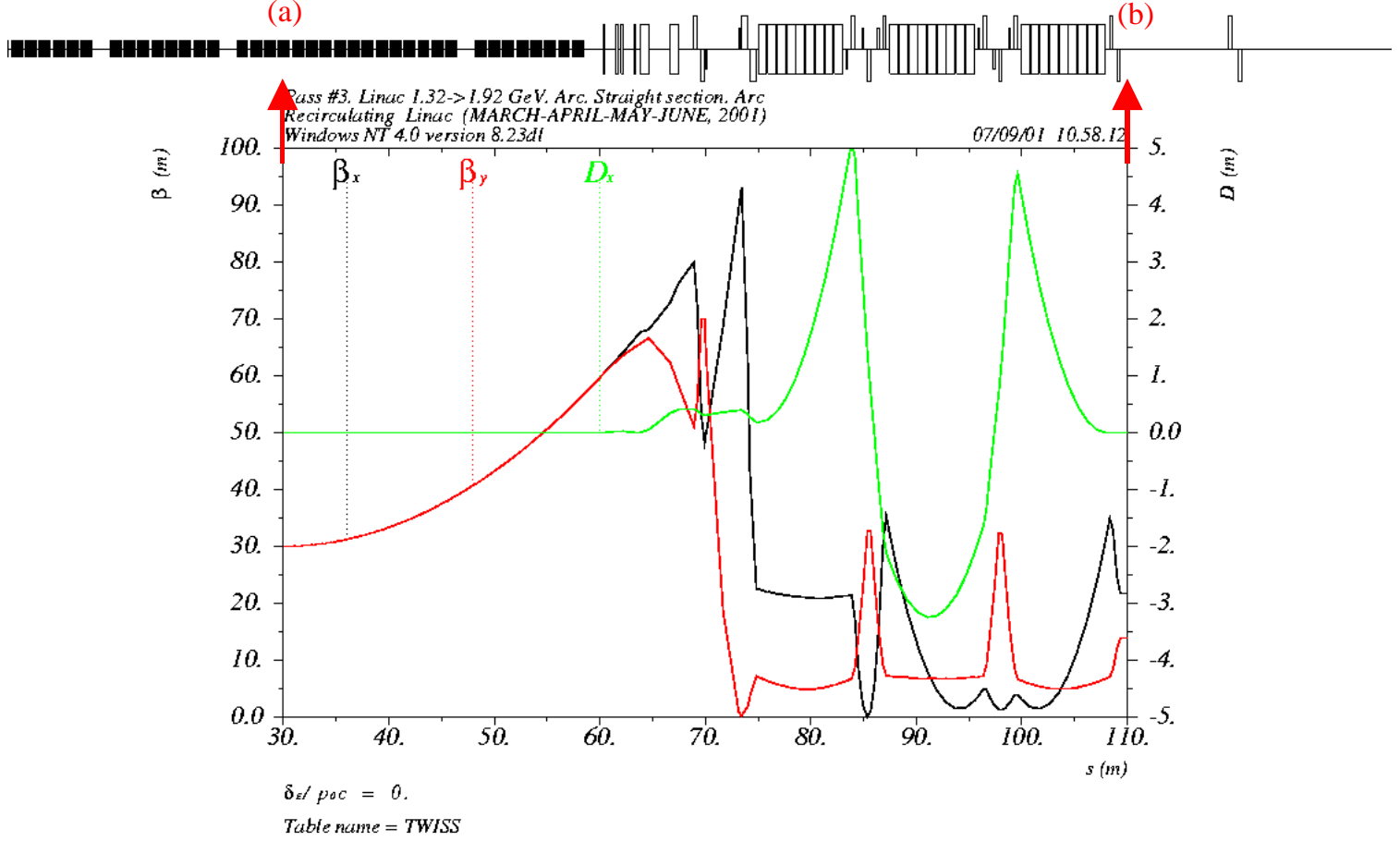

Figure 4. Lattice functions from the center of the main linac (a), through the end of $\operatorname{arc} 3(\mathrm{~b})(1.9 \mathrm{GeV})$.

The lattice of the photon production section consists of six identical cells with matching sections on each side. It contains seven two-meter long straight sections for insertion devices and six two-Tesla magnetic field dipole magnets located between insertion devices. At the beginning and end of the photon production section, we use rf cavities operating in the first dipole mode $\left(\mathrm{TM}_{110}\right)$. These prepare the electron beam to allow x-ray compression from $\sim 1$ ps to $\sim 50$ fs [ZHO99]. The first cavity provides a time dependent transverse (vertical) kick to the electron beam that the second cavity cancels. The rf phase is adjusted so that the kick acts in opposite directions on the head and the tail of the bunches, with no perturbation at the center of the bunch. The electrons perform betatron oscillations after the kick with vertical betatron phase advance of $n \pi$ between the center of the rf cavity and the center of the $n$-th undulator. The vertical betatron phase advance is $n \pi / 2$ between the center of the undulator and the center of the adjacent $2 \mathrm{~T}$ dipole magnet. Therefore within a bending magnet the electrons in a bunch have zero angle and a transverse displacement variation along the bunch described by:

$$
\delta y(z)=\frac{e U}{E_{\text {beam }}} \sqrt{\beta_{\text {cavity }} \beta_{\text {bend }}} \sin \left(k_{r f} z\right)
$$

where $\beta_{\text {cavity }}$ is the beta-function in the cavity, $\beta_{\text {bend }}$ is the beta-function in the bend magnet, $e U$ is the energy gain at the maximum of the RF field, $k_{r f}$ is the rf wave number, $z$ is the electron position relative to the bunch center, and $E_{\text {beam }}$ the electron beam energy. Similarly, we find in the insertion devices that the electrons within a bunch have no transverse displacement and angular variation along the bunch described by:

$$
\delta y^{\prime}(z)=\frac{e U}{E_{\text {beam }}} \sqrt{\frac{\beta_{\text {cavity }}}{\beta_{I D}}} \sin \left(k_{r f} z\right)
$$


where $\beta_{I D}$ is the beta-function in the insertion device. Consequently, the photon production bend magnets are $\mathrm{x}$-ray sources with a vertical position-time correlation and the insertion devices are $\mathrm{x}$-ray sources with an angular-time correlation. We discuss in Section 3 how such correlations are exploited to compress $\mathrm{x}$-ray pulses. The present design has $\beta_{\text {cavity }}=50 \mathrm{~m}, \beta_{I D}=1 \mathrm{~m}$, and $\beta_{\text {bend }}=3 \mathrm{~m}$, while it maintains the ability to vary these values over a wide range. Figure 5 shows lattice functions from the end of arc 4 and through the first two photon production section lattice cells.

The straight sections connecting the arcs have a simple FODO focusing structure that is used for matching of beta-functions between the arcs. They may also be used for independent adjustments of the betatron phase advances on each beam orbit.

As a result of the relatively low electron beam energy the effect of synchrotron radiation on the electron beam is small. At $2.5 \mathrm{GeV}$ the average energy loss of electrons in the photon production section is only $500 \mathrm{keV}$ and the increase of the energy spread due to quantum fluctuations of synchrotron radiation is $\sim 50 \mathrm{keV}$, less than the beam energy spread of $150 \mathrm{keV}$.

Major parameters of the present machine concept are presented in Table 1.

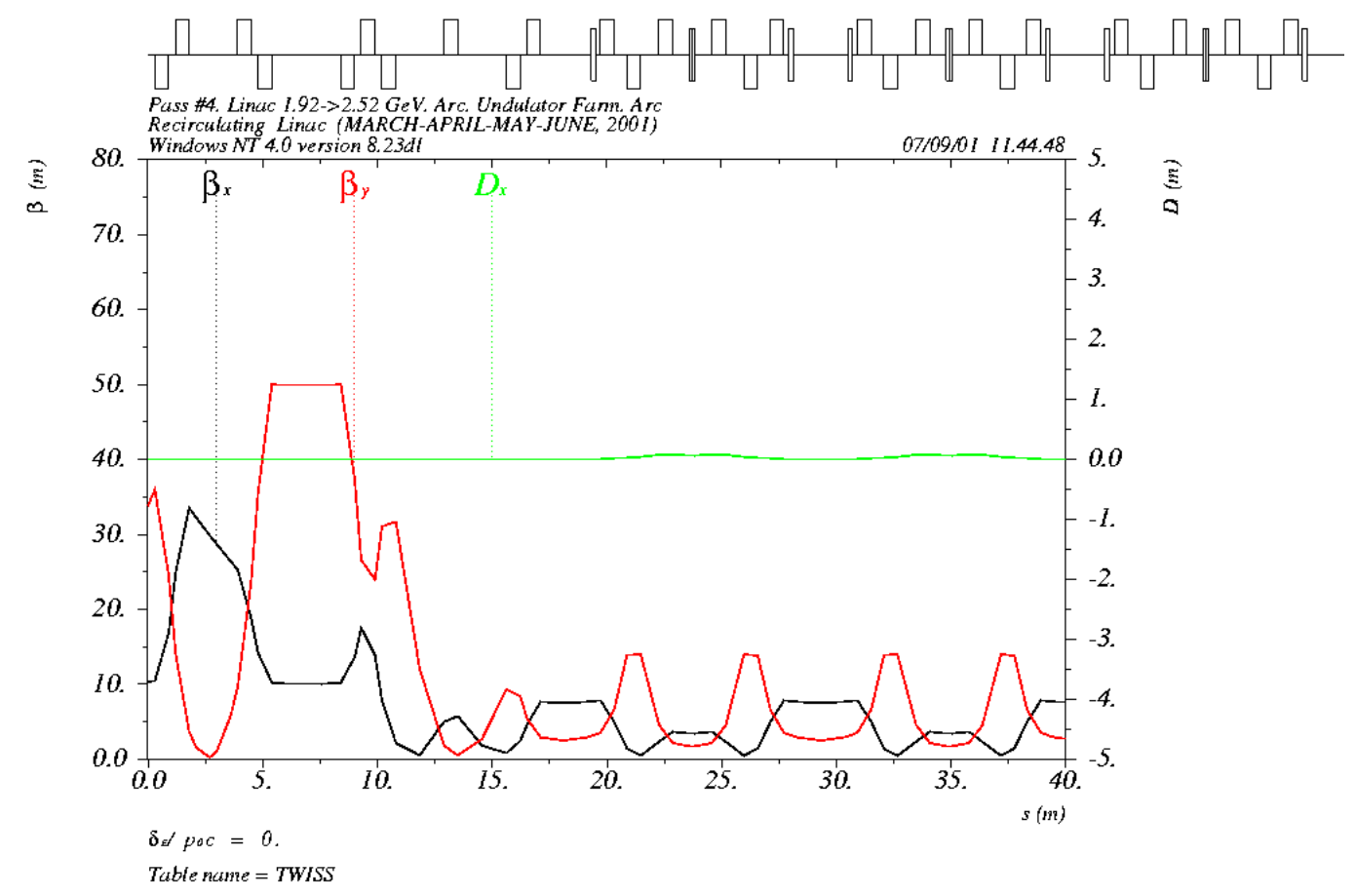

Figure 5. Lattice functions from the end of arc 4 through the second undulator cell of the photon production section. 
Table 1. Principal machine parameters

\begin{tabular}{|l|l|}
\hline Bunch charge & $1 \mathrm{nC}$ \\
\hline Bunch repetition rate & $10^{4} \mathrm{~Hz}$ \\
\hline Beam energy spread at $2.5 \mathrm{GeV}$ & $200 \mathrm{keV}$ \\
\hline Bunch length in photon production section & $1 \mathrm{ps}$ (full length) \\
\hline Electron tilt angle in the undulator & $0.14 \mathrm{mrad}$ \\
\hline Bunch angle in dipoles & $68^{\circ}$ \\
\hline RF deflection cavity frequency & $2.6 \mathrm{GHz}$ \\
\hline RF deflection cavity transverse voltage & $9.2 \mathrm{MV}$ \\
\hline Undulator length & $2 \mathrm{~m}$ \\
\hline Field in a bend magnet radiation source & $2 \mathrm{~T}$ \\
\hline Bunch length at gun exit & $10 \mathrm{ps}$ \\
\hline Bunch length at first pass through linac & $5 \mathrm{ps}$ \\
\hline $\mathrm{x} / \mathrm{y}$ normalized emittance at gun & $20 / 0.4 \quad 10^{-6} \mathrm{~m}-\mathrm{rad}$ \\
\hline Beam energy spread at gun & $15 \mathrm{keV}$ \\
\hline $\mathrm{x} / \mathrm{y}$ emittance in photon production section & $4 / 0.0810^{-9} \mathrm{~m}-\mathrm{rad}$ \\
\hline $\mathrm{x} / \mathrm{y}$ beam size in photon production section & $100 / 8.5 \mu \mathrm{m}$ \\
\hline $\mathrm{x} / \mathrm{y}$ beam divergence in photon production section & $40 / 8.5 \mu-\mathrm{rad}$ \\
\hline Energy gain per pass & $600 \mathrm{MeV}$ \\
\hline RF frequency & $1.3 \mathrm{GHz}$ \\
\hline RF klystron power & $1 \mathrm{MW}$ \\
\hline
\end{tabular}

\section{Ultra-fast X-ray Pulse Production}

The photon production section of the recirculating linac will provide two classes of radiation sources: bending magnets, and undulators. In the bend magnets, electrons will have a vertical position-time correlation, in the undulators, a vertical angle-time correlation. Both sources will produce x-ray radiation that can be compressed in the x-ray beamline to an X-ray pulse length of $\sigma_{x-\text { ray }} \sim 7-10 \mu \mathrm{m}$.

Asymmetrically cut crystals may be used as optical elements in the x-ray pulse compression scheme [MAT93, ZH099]. As a result of the different angles of incidence and diffraction, a crystal may be oriented to produce a variable path length across the x-ray beam as shown in Figure 6. After the collection optics, two asymmetrically cut crystals can be used as both monochromator and pulse compressor for the xrays. The optical path length difference $\Delta l$ for two asymmetrically cut crystals is given by:

$$
\Delta l=2 \frac{\Delta y}{\sin (\theta-\alpha)}(\sin \theta \sin \alpha) \frac{\sin (\theta+\alpha)}{\sin (\theta-\alpha)}
$$

where $\Delta y$ is the vertical height of the beam at the crystals, $\theta$ is the Bragg angle, $\alpha$ is the asymmetry angle between the Bragg planes and the crystal surface. A practical example of x-ray pulse compression for $1.5 \AA$ wavelength is given in Table 2 . 


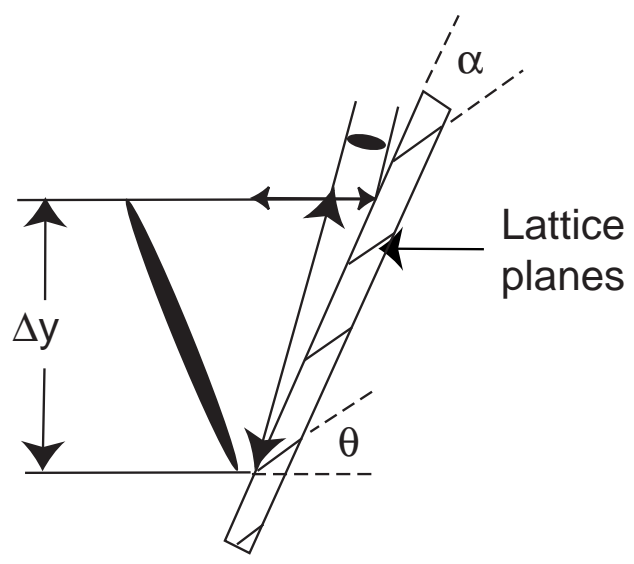

Figure 6. Asymmetrically cut crystal with variable optical path length.

Table 2. Parameters of asymmetrically cut crystals for x-ray pulse compression.

\begin{tabular}{|c|c|c|c|c|c|}
\hline & $\lambda$ & $\Delta y$ & $\theta$ & $\alpha$ & $\Delta l$ \\
\hline $\operatorname{Si}(220)$ & $1.5 \AA$ & $1.4 \mathrm{~mm}$ & $22.325^{\circ}$ & $-4.3^{\circ}$ & $0.3 \mathrm{~mm}$ \\
\hline
\end{tabular}

For a bending magnet source, with a vertical position-time correlation, the collection optics may consist of a telescope with two vertically focusing mirrors which produce a magnified image of the source at the asymmetrically cut crystals. The magnification of the telescope is set to 5 .

For the undulator source, with angle-time correlation, a single collimating mirror is sufficient for the collection optics. The distance from the undulator source the first mirror is short, approximately $3 \mathrm{~m}$, but this is feasible given the low average beam current of the accelerator.

As seen in the Eq. 3, the magnitude of the X-ray pulse compression depends upon the Bragg angle $\theta$. Additional rotation of the crystals about the normal to the Bragg planes, $\phi$, varies the crystal asymmetry in the diffraction plane, allowing tuning of the monochromator photon energy while keeping the $\mathrm{x}$-ray pulse compression fixed [SMI94]. Kirkpatrick-Baez mirrors can be used to refocus the x-rays onto the samples after the crystal.

Alternatively, one can use the angle-time or coordinate-time correlation of the radiation for observation at different time delays. This can be done without compressing the $\mathrm{x}$-ray pulse by detecting the individual time slices on a position sensitive detector after the sample. For this technique to be effective, the angle-time correlation of the incident $\mathrm{x}$-rays must be preserved after the sample.

To reach an $\mathrm{x}$-ray pulse length of $\sigma_{\mathrm{x} \text {-ray }} \sim 7-10 \mu \mathrm{m}$ we require a rf deflection voltage defined by one of two equations [ZH099]:

$$
\frac{e U}{E_{\text {beam }}} \geq \frac{1}{k_{r f} \sigma_{x-\text { ray }}} \sigma_{y}^{r f} \cdot \sqrt{1+\left(\frac{\sigma_{r}}{\sigma_{y}}\right)^{2}}
$$




$$
\frac{e U}{E_{\text {beam }}} \geq \frac{1}{k_{r f} \sigma_{x-\text { ray }}} \sigma_{y^{\prime}} \sqrt{1+\left(\frac{\sigma_{r^{\prime}}}{\sigma_{y^{\prime}}}\right)^{2}}
$$

where $\sigma_{r}=\frac{\lambda_{x-r a y}}{4 \pi \sigma_{r^{\prime}}}$

$k_{r f}$ is the rf wavenumber, $\sigma_{y^{\prime}}^{f}$ is the vertical angular size of the electron beam in the rf deflecting cavity, $\sigma_{y}$ is the vertical electron beam size in the bending magnet, $\sigma_{y^{\prime}}$ is the vertical angular beam size in the undulator, $\sigma_{r^{\prime}}$ is the opening angle of the radiation, $\sigma_{r}$ is the diffraction-limited size of the radiation, and $\lambda_{x-r a y}$ is the xray wavelength. Using in Eqns. ( $4 \mathrm{a}, \mathrm{b})$ with $E_{\text {beam }}=2.5 \mathrm{GeV}$, a normalized vertical emittance of $0.35 \mathrm{~mm}$ $\operatorname{mrad}, k_{r f}=7.32 \mathrm{~cm}^{-1}\left(F_{r f}=2.6 \mathrm{GHz}\right), \lambda_{x-r a y}=1.5 \AA$, and $e U=9.2 \mathrm{MeV}$, we calculate the FWHM duration of the compressed $\mathrm{x}$-ray pulse shown in Figure 7 as a function of the $\mathrm{x}$-ray photon energy. At larger photon energies the pulse duration is limited by the vertical beam emittance, while at smaller photon energies it is mainly defined by the opening angle of the radiation or by the diffraction-limited size of the radiation.

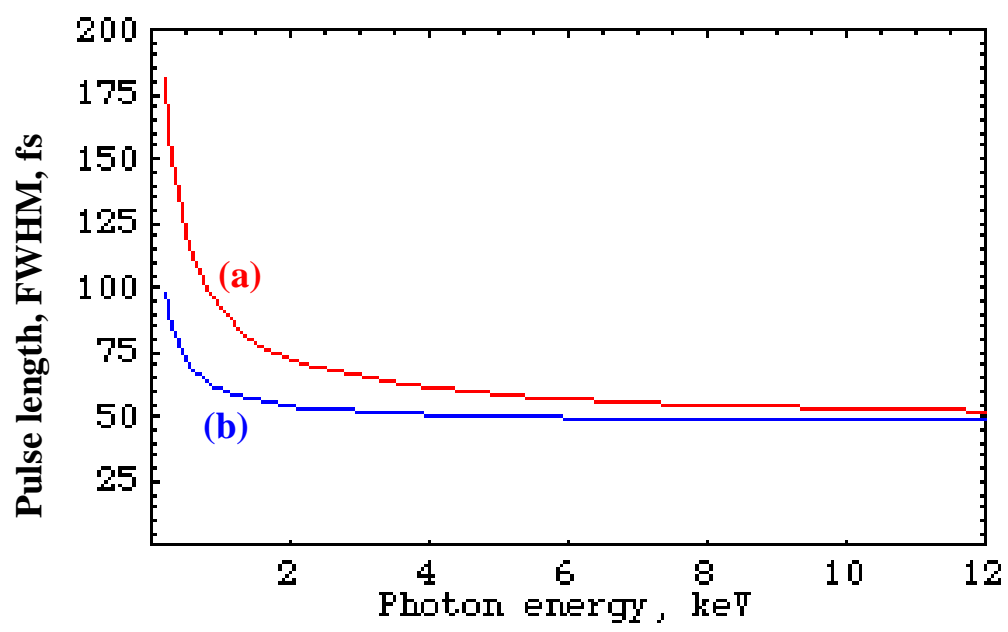

Figure 7. Duration of the compressed $\mathrm{x}$-ray pulse as a function of the $\mathrm{x}$-ray photon energy for (a) an undulator source, (b) a bend magnet source.

\section{Flat Electron Beam Production}

Our approach for producing an ultra-short x-ray pulse relies on the availability of an electron beam with a flat aspect ratio so that one transverse dimension ( $y$, vertical) is small. In a circular storage ring, this aspect ratio is common based on the desired synchrotron radiation emission characteristics and the planar design of the machine that makes dominantly horizontal dispersion desirable. In a recirculating linac, the radiation damping process that allows this large aspect ratio is cut short and the best one can do is essentially maintain the emittance from the injector through the rest of the machine without degradation. Thus, we require that electrons be produced with the proper emittance and aspect ratio before being injected into the recirculating linac. 
A method of producing a circular cross section beam from the nominal flat-beam of a storage ring was conceived by Derbenev in order to facilitate electron beam cooling in hadron colliders [DER98]. Later the process was inverted to produce a flat beam from a circular beam originating from a cathode [BRI99] and it is by using this technique that we propose to provide a large $x / y$ emittance ratio. The key lies in the presence of a longitudinal solenoidal field at the cathode (typically avoided by the use of magnetic bucking coils). The beam emitted from the cathode surface propagates along the magnetic field lines until particles produced off-axis acquire angular deflections when leaving the solenoidal field. Propagating the beam through a quadrupole channel with an identity transform in the $x$-direction and $90^{\circ}$ phase advance in the $y$-direction, and with a suitable choice of solenoid field strength such that the symmetry of the $x-y$ motion is broken, results in a flat beam inclined at $45^{\circ}$. A suitably constructed skew-quadrupole channel then allows production of a flat beam aligned in either the horizontal or vertical plane. This principle has been successfully demonstrated at the Fermilab A0 photo-injector test facility [EDW00], and the set-up for flatbeam production at the Fermilab A0 facility is shown in Figure 8. An emittance ratio of order $10^{2}$ may be achieved by this procedure, with a normalized emittance of $\gamma\left(\varepsilon_{x} \times \varepsilon_{y}\right)^{0.5} \approx 10^{-6} \mathrm{~m}$-rad at the cathode for a $1 \mathrm{nC}$ bunch [BRI99], however these estimates are limited to low charge without space charge effects, within the flat aspect ratio part of the beamline.

Typical operational parameters for the A0 facility are shown in Table 3. The lower portion of Figure 8 shows images from the demonstration of flat beam production, with a round beam exiting the superconducting multi-cell cavity (left image), and a vertically oriented flat beam at the exit of the quadrupole channel (right image). An emittance ratio of 50:1 has been measured on a $1 \mathrm{nC}$ bunch with measured normalized horizontal and vertical emittances of $\gamma \varepsilon_{x}=0.9 \times 10^{-6} \mathrm{~m}$-rad, $\gamma \varepsilon_{\mathrm{y}}=45 \times 10^{-6} \mathrm{~m}$-rad.

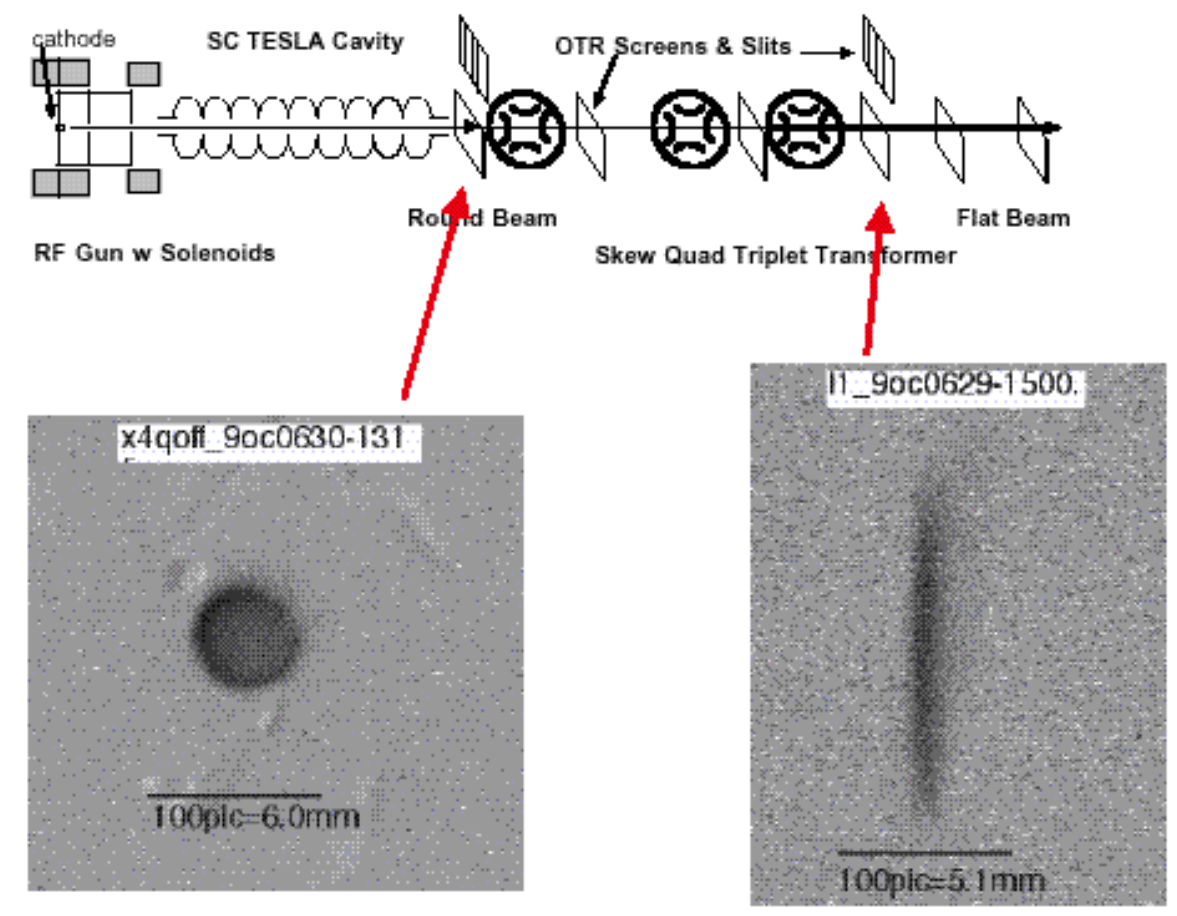

Figure 8. Flat beam production at the Fermilab A0 test facility. Upper part shows the facility layout, lower part shows measured beam images at the exit of the superconducting cavity (left, round beam), and after the transformation in the quadrupole channel (right, flat beam vertically oriented). 
Table 3. A0 facility typical parameters

\begin{tabular}{|l|l|}
\hline Cathode & $\mathrm{Cs}_{2} \mathrm{Te}$ \\
\hline Cathode quantum efficiency & $\sim 1-2 \%$ \\
\hline Laser & $\begin{array}{l}\text { Mode-locked Nd:YLF, 25 } \mu \mathrm{J} \text { at } 1054 \mathrm{~nm} \\
\text { Nd:glass multipass amplifier } \\
\text { Two-stage harmonic generation to 263 nm }\end{array}$ \\
\hline Laser UV energy & $1-5 \mu \mathrm{J}$ per bunch \\
\hline Laser pulse length & $1.9 \mathrm{ps}$ (unstacked), 11 ps (stacked) \\
\hline Laser spot size on cathode (rms) & $0.7-1.6 \mathrm{~mm}$ \\
\hline Gradient at cathode & $35-40 \mathrm{MVm} \mathrm{M}^{-1}$ \\
\hline Gun solenoid strength & $1200 \mathrm{Gauss}$ typical \\
\hline Charge & $1-10 \mathrm{nC}$ \\
\hline Gradient in 9-cell accelerating module & $12 \mathrm{MVm}-1$ \\
\hline Electron energy after gun & $4.5-5.0 \mathrm{MeV}$ \\
\hline Electron energy after accelerating module & $17-18 \mathrm{MeV}$ \\
\hline Typical pulse structure & $10-20 \mathrm{bunches}, 1 \mu \mathrm{s}$ bunch spacing typical \\
\hline Rf pulse structure & $30-600 \mu \mathrm{s} @ 1 \mathrm{~Hz}$ \\
\hline Normalized emittance $\gamma \varepsilon$ & $3.7 \mathrm{x} 10^{-6} \mathrm{~m}-\mathrm{rad} @ 1 \mathrm{nC}$ \\
\hline Momentum spread $\sigma p / \mathrm{p}$ & $0.25-0.38 \%$ \\
\hline Bunch length (uncompressed) & $1.6 \mathrm{~mm} \mathrm{@} 1 \mathrm{nC}$ \\
\hline Peak current & $75-330 \mathrm{~A}$ \\
\hline
\end{tabular}

\section{RF Photocathode Gun}

Modern rf photocathode guns are capable of producing electron beams exceeding the brightness of third generation light sources by more than two orders of magnitude. Such high brightness is achieved by generating the electrons in the presence of a strong electric field that rapidly accelerates the particles and thus reduces emittance growth from space charge effects that dominate at low energy. Major parameters for the rf gun are shown in Table 4, and are consistent with measured values at existing facilities [PER01].

Conventionally, photocathode if guns employ a half-length pillbox cell for the cathode cavity followed by a full cell for rapid acceleration of emitted electrons. These have been shown to produce good emittance at high electric field gradient when operating at a low duty factor. For CW or high duty factor operation, thermal limitations may prevent such a design from operating at sufficiently high gradient. Typical rf guns operate with accelerating fields of approximately $50 \mathrm{MVm}^{-1}$, and low repetition rates of the order of $10 \mathrm{~Hz}$. Our design requires a much higher bunch rate of order $10 \mathrm{kHz}$.

The increased repetition rate significantly increases the heat deposited by rf currents on the cavity surfaces. We have produced a conceptual design with optimized cavity geometry to allow cooling of the cavity surfaces, and operation at high gradient and high repetition rate. In this design the first cell is modified by the inclusion of a re-entrant nose-cone, on the end face of which the photo-cathode is mounted. This nose-cone serves two purposes: it increases surface area to reduce deposited power density, and it enhances the accelerating electric field at the cathode. The cavity profile (cylindrically symmetric, beam is along the $\mathrm{x}$-axis) and $\mathrm{E}$ and $\mathrm{H}$ fields are shown in Figure 9. 
Table 4. Rf gun requirements

\begin{tabular}{|l|l|}
\hline Energy & $10 \mathrm{MeV}$ \\
\hline Rf frequency & $1.3 \mathrm{GHz}$ \\
\hline Peak accelerating field at cathode & $60 \mathrm{MVm}^{-1}$ \\
\hline Bunch charge & $1 \mathrm{nC}$ \\
\hline Laser spot size on cathode (rms) & $\sim 1 \mathrm{~mm}$ \\
\hline Normalized emittance $\gamma \varepsilon$ & $\sim 1 \times 10^{-6} \mathrm{~m}-\mathrm{rad}$ \\
\hline Peak current & $100 \mathrm{~A}$ \\
\hline Bunch length & $10 \mathrm{ps}$ \\
\hline Energy spread & $15 \mathrm{keV}$ \\
\hline Laser wavelength & $267 \mathrm{~nm}$ \\
\hline Laser pulse energy & $100 \mu \mathrm{J}$ \\
\hline Laser repetition rate & $\sim 10 \mathrm{kHz}$ \\
\hline
\end{tabular}
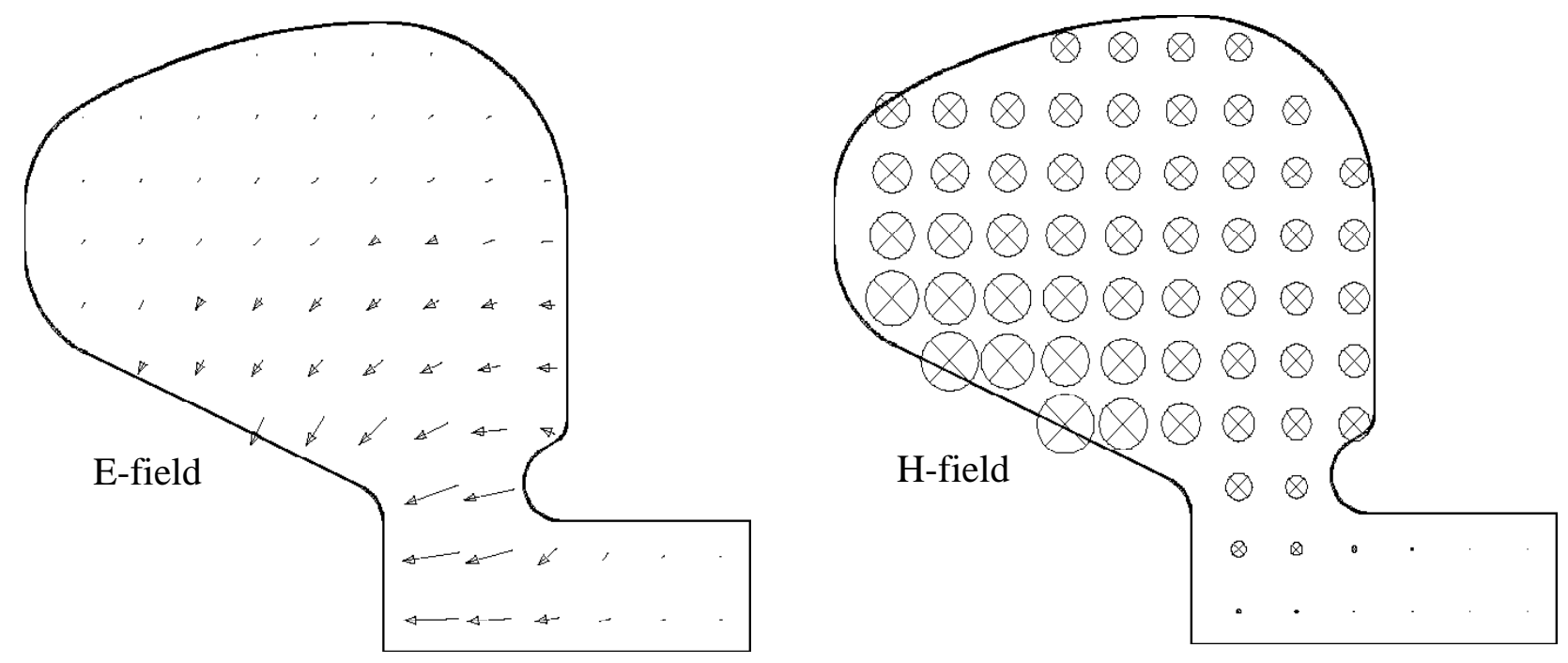

Figure 9. Cross-section of high-power rf gun first cell. The figure shows the upper section of a cylindrically symmetric structure, with the lower horizontal line the symmetry axis (beam axis). 
Table 5. Parameters of reentrant and pillbox rf gun cavity models.

\begin{tabular}{|c|c|c|c|c|c|c|c|c|}
\hline $\begin{array}{c}\text { Gun frequency } \\
(\mathrm{MHz})\end{array}$ & $\begin{array}{l}\text { E-field at } \\
\text { cathode } \\
\left(\mathrm{MVm}^{-1}\right)\end{array}$ & $\begin{array}{c}\text { Peak } \\
\text { RF } \\
\text { power } \\
(\mathrm{kW}) \\
\end{array}$ & $\begin{array}{c}\text { Coupling } \\
\text { (ß) }\end{array}$ & $\begin{array}{c}\text { Klystron } \\
\text { power } \\
(\mathrm{kW})\end{array}$ & $\begin{array}{l}\text { Repetition } \\
\text { rate }(\mathrm{kHz})\end{array}$ & $\begin{array}{c}\text { Duty } \\
\text { factor } \\
\text { (for 3- } \tau \\
\text { fill) } \\
\end{array}$ & $\begin{array}{c}\text { Average } \\
\text { power } \\
\text { dissipated } \\
(\mathrm{kW}) \\
\end{array}$ & $\begin{array}{l}\text { Maximum power } \\
\text { density on cavity } \\
\text { surface }\left(\mathrm{Wcm}^{-2}\right)\end{array}$ \\
\hline \multirow[t]{3}{*}{476} & 16.7 & 100.7 & 1 & 100.7 & $\mathrm{CW}$ & 1 & 100.71 & 99.0 \\
\hline & 35.0 & 442.4 & 1 & 442.4 & 10 & 0.228 & 100.67 & 98.9 \\
\hline & 22.6 & 184.4 & 7.4 & 439.7 & 100 & 0.542 & 99.94 & 98.2 \\
\hline \multirow[t]{3}{*}{1300} & 13.8 & 13.6 & 1 & 13.6 & $\mathrm{CW}$ & 1 & 13.62 & 99.0 \\
\hline & 62.0 & 274.9 & 1 & 274.9 & 10 & 0.050 & 13.74 & 99.9 \\
\hline & 40.1 & 115.0 & 7.4 & 274.2 & 100 & 0.119 & 13.68 & 99.5 \\
\hline \multirow[t]{3}{*}{2600} & 11.0 & 3.4 & 1 & 3.4 & $\mathrm{CW}$ & 1 & 3.41 & 99.7 \\
\hline & 82.6 & 192.3 & 1 & 192.3 & 10 & 0.018 & 3.41 & 99.8 \\
\hline & 53.5 & 80.7 & 7.4 & 192.3 & 100 & 0.042 & 3.41 & 99.7 \\
\hline \multirow[t]{3}{*}{ Pillbox 1300} & 13.8 & 129.0 & 1 & 129 & $\mathrm{CW}$ & 1 & 129 & 207 \\
\hline & 37.8 & 968.0 & 1 & 968 & 10 & 0.064 & 62 & 100 \\
\hline & 24.6 & 408.0 & 7.4 & 973 & 100 & 0.152 & 62 & 100 \\
\hline
\end{tabular}

By scaling the geometry of this design, we have produced models which operate at different frequencies: $476 \mathrm{MHz}, 1.3 \mathrm{GHz}$, and $2.6 \mathrm{GHz}$. Table 5 lists parameters for these cavities calculated using the URMEL code for different pulse repetition rates. A $1.3 \mathrm{GHz}$ pillbox cavity for comparison. Note that the average power dissipated for a $1.3 \mathrm{GHz}$ pillbox cavity is a factor 4.5 to 9.5 larger than the nose-cone geometry, depending upon repetition frequency. In this comparison the maximum power density dissipated by rf currents in the cavity walls is limited to $100 \mathrm{Wcm}^{-2}$ to allow for adequate cooling by water channels, and acceptable stress levels in the copper cavity.
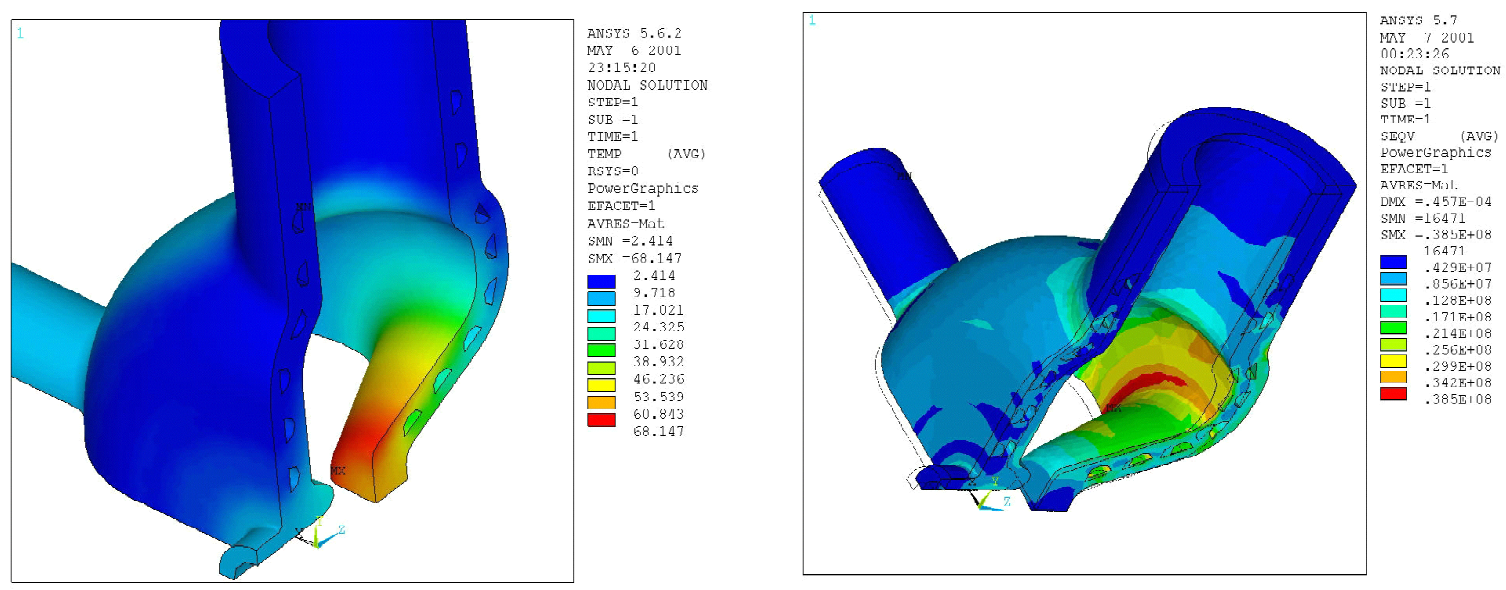

Figure 10. ANSYS calculation of temperature rise over ambient (left), and Von Mises stresses (right) in a $1.3 \mathrm{GHz}$ cavity, and for $60 \mathrm{MVm}^{-1}$ accelerating gradient, at $10 \mathrm{kHz}$. 
The mechanical behavior of the cavity has been modeled using the ANSYS finite-element code. With the cavity geometry as input, the resonant mode fields were determined using an electromagnetic module of the ANSYS code. Surface currents are then determined, and power density from the resistive losses in the cavity skin-depth. Specifying a maximum power density of $100 \mathrm{Wcm}^{-2}$ then dictates the maximum field values in the structure. To reduce the temperature rise and stress in the cavity, water cooling channels are included in the cavity shell and reentrant nosecone.

From these calculations, an acceptable accelerating gradient of $40 \mathrm{MVm}^{-1}$ or greater is achieved even for repetition rates as high as $100 \mathrm{kHz}$. For the $1.3 \mathrm{GHz}$ cavity at $10 \mathrm{kHz}$ repetition rate, $60 \mathrm{MVm}^{-1}$ accelerating gradient, the maximum temperature rise is limited to $68^{\circ} \mathrm{C}$, as shown in Figure 10 (left). The corresponding thermal stresses of up to $38.5 \mathrm{Mpa}$ (approximately 5,500 psi) are well within the limits of approximately 18,000 psi for $10^{4}$ cycles in a copper structure. Figure 10 also shows the Von Mises stresses calculated for the same conditions (right). Note that the cavity is over coupled at $100 \mathrm{kHz}$ to allow a shorter fill time, and the accelerating gradient is reduced to maintain the surface power density limit.

\section{RF Systems}

\subsection{Linacs}

Our linac design is based on superconducting rf technology developed for the TESLA project [TES01]. We propose to use modules similar to those designed for the TESLA FEL injector to take advantage of the developments made by the TESLA collaboration in obtaining high gradient accelerating cavities, and to avoid an expensive and time-consuming independent R\&D program. The TESLA rf cavity parameters are well suited to our requirements, and many complex issues including higher-order mode damping, suppression of multipacting, cavity materials preparation and fabrication, input power coupler design, have been studied and solutions explored.

Figure 11 shows the cross-section schematic view of a TESLA 9-cell superconducting rf (scrf) cavity [TES01]. Eight such cavities are integrated with cryogenics and other systems into a TESLA FEL injector linac cryomodule, with cross-section (end view) shown in Figure 12 [TES01]. Each of these cryomodules is approximately $12 \mathrm{~m}$ long, and we require four such cryomodules to achieve an energy gain of $\geq 600 \mathrm{MeV}$ per pass. Operating at the TESLA gradient of $23 \mathrm{MVm}^{-1}$ would allow a final beam energy of $\sim 3 \mathrm{GeV}$, but the lower gradient provides a design margin and reduced power dissipation in the cryostat. We will operate slightly off-crest of the rf field maximum and our design considerations here are for a peak accelerating gradient of $20 \mathrm{MVm}^{-1}$. An additional identical cryomodule is used for the injector linac.

The superconducting cavities have a high quality factor $\left(\mathrm{Q}_{0} \sim 10^{10}\right)$, resulting in long filling times. Typically, power is applied to standing wave cavities for three filling times before the beam enters the structure. This allows time for energy to build up in the cavity so that the required voltage can be developed. Since we wish to operate at a bunch rate in excess of $10 \mathrm{kHz}$, we will not be able to change the power in the cavity at a rate commensurate with the bunch time structure, and we must operate the linac rf system in continuous wave $(\mathrm{CW})$ mode. This, of course, increases the power requirements of the system, both in terms of the rf drive power, and the cryogenic system power, which is needed to absorb resistive losses in the cavities. The beam current of only $0.04 \mathrm{~mA}$ in the main linac cavities during the acceleration cycle does not produce significant beam loading. 


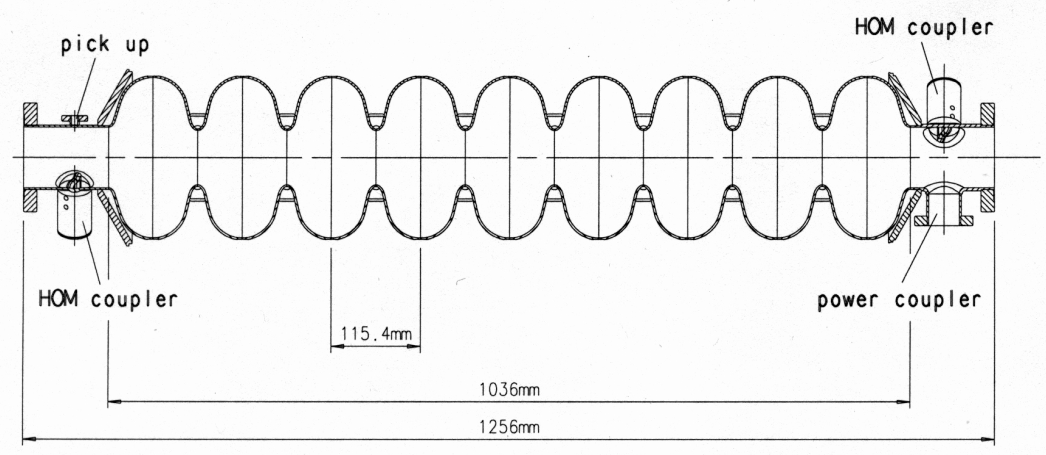

Figure 11. TESLA 9-cell superconducting $1.3 \mathrm{GHz}$ cavity longitudinal cross-section.

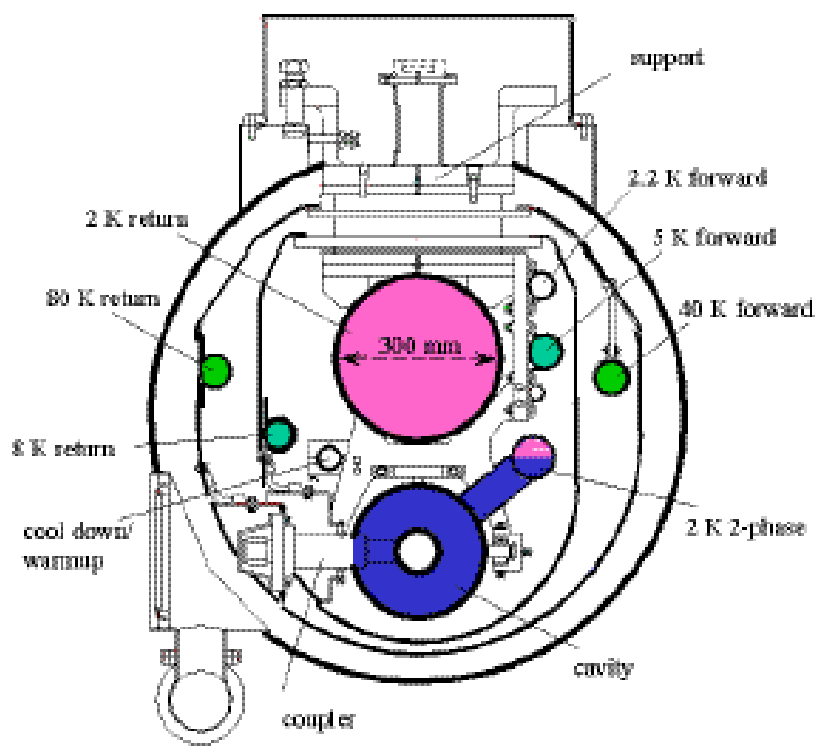

Figure 12. TESLA cryomodule end view cross-section.

Typically, rf systems are designed such that reflected power from the accelerating structures is minimized, and a cavity coupling factor of approximately 20 would provide the most efficient use of $\mathrm{rf}$ power. The requirement to provide a cavity bandwidth sufficiently large to allow for cavity frequency tuning, however, stipulates a significantly larger coupling factor. A conservative cavity bandwidth of 200 $\mathrm{Hz}$ has been chosen in order to allow feedback control of the resonant frequency. The externally loaded quality factor, $Q_{\text {external }}$, may be expressed as:

$$
Q_{\text {external }}=\frac{F_{\text {resonant }}}{\Delta F}=\frac{1.3 \times 10^{9}}{200}=6.5 \times 10^{6}
$$


The cavity coupling factor, $\beta$, is the ratio of power loss in the external circuit to the power loss in the cavity. With an unloaded $Q$ value of $10^{10}$, this externally loaded quality factor results in a large coupling factor of the drive power transmission line to the cavity:

$$
\beta=\frac{Q_{\text {unloaded }}}{Q_{\text {external }}}=\frac{1 \times 10^{10}}{6.5 \times 10^{6}}=1.54 \times 10^{3}
$$

The strongly over-coupled cavities reflect most of the incident drive power. This results in a large requirement for $\mathrm{rf}$ power in order to produce the specified accelerating voltage, as follows from considerations below:

$$
\begin{gathered}
\frac{P_{\text {reflected }}}{P_{\text {incident }}}=\left(\frac{\beta-1}{\beta+1}\right)^{2}=\left(\frac{1539}{1541}\right)^{2}=0.9974 \\
P_{\text {cavity }}=P_{\text {incident }}-P_{\text {reflected }}=P_{\text {incident }}\left(1-\left(\frac{\beta-1}{\beta+1}\right)^{2}\right)=P_{\text {incident }} \times 2.59 \times 10^{-3}
\end{gathered}
$$

where $P_{\text {incident }}$ is the rf power incident at the cavities, $P_{\text {reflected }}$ is the rf power reflected from the cavity, and $P_{\text {cavity }}$ the power dissipated in the cavity walls. $P_{\text {cavity }}$ is related to the $R / Q$ and the accelerating voltage $V$ by:

$$
P_{\text {cavity }}=\frac{V^{2}}{\frac{R}{Q} Q}=\frac{\left(20 \times 10^{6} \times 1.038\right)^{2}}{1036 \times 10^{10}}=42 \mathrm{~W}
$$

We find that the incident power required to produce $20 \mathrm{MVm}^{-1}$ in the main linac is then $16 \mathrm{~kW}$ per cavity, and to produce $15 \mathrm{MVm}^{-1}$ in the injector linac is $9 \mathrm{~kW}$ per cavity. With four main linac cryomodules plus one injector cryomodule, and an additional $25 \mathrm{~kW}$ for beam power, we require a minimum rf power of $610 \mathrm{~kW}$. Operating at the TESLA cavity design gradient of $23 \mathrm{MVm}^{-1}$ would require $55 \mathrm{~W}$ dissipated per main linac cavity, and $780 \mathrm{~kW}$ total rf power. To accommodate flexible operations, allow for losses in transmission lines, overhead for stable feedback operations, and additional power for the rf photocathode gun, we consider $1 \mathrm{MW}$ total rf power systems. Assuming an efficiency of $65 \%$ for a klystron and its power supply, we require a wall-plug power of $1.5 \mathrm{MW}$ for the rf systems of the main linac, rf gun, and injector linac.

Linac system $\mathrm{rf}$ requirements are summarized in Table 6. Power recovered by recirculating the beam in energy recovery mode gains at most $25 \mathrm{~kW}$, a small part of the total $\mathrm{rf}$ system requirements. 
Table 6. Linac RF system requirements

\begin{tabular}{|l|l|}
\hline Energy gain per pass main linac & $600 \mathrm{MeV}$ \\
\hline Energy gain per pass injection linac & $110 \mathrm{MeV}$ \\
\hline $\mathrm{E}_{\text {accelerating }}$ main linac & $20 \mathrm{MVm}^{-1}$ \\
\hline $\mathrm{E}_{\text {accelerating injector linac }}$ & $15 \mathrm{MVm}^{-1}$ \\
\hline Operating mode & $\mathrm{CW}$ \\
\hline Dynamic power losses per main linac cavity & $42 \mathrm{~W}$ \\
\hline Dynamic power losses per injector linac cavity & $24 \mathrm{~W}$ \\
\hline Average beam current (four main linac passes) & $0.04 \mathrm{~mA}$ \\
\hline Beam power requirement & $25 \mathrm{~kW}$ \\
\hline Qunloaded & $1 \times 10^{10}$ \\
\hline Qexternal & $6.5 \times 10^{6}$ \\
\hline Cavity R/Q (P=V'/R definition) & $1036 \Omega$ \\
\hline Cavity resonance bandwidth & $200 \mathrm{~Hz}$ \\
\hline Cavity coupling factor & $1.54 \times 10^{3}$ \\
\hline Length of cavity & $1.038 \mathrm{~m}$ \\
\hline Rf losses per main linac cryomodule & $336 \mathrm{~W}$ \\
\hline Rf power requirement per main linac cryomodule & $128 \mathrm{~kW}$ \\
\hline Total accelerator systems rf power & $1 \mathrm{MW}$ \\
\hline AC power for rf systems & $1.5 \mathrm{MW}$ \\
\hline
\end{tabular}

\subsection{RF Deflecting Cavities}

Cavities operating in the $\mathrm{TM}_{110}$ mode are required to provide the transverse (vertical) head-tail kick to each bunch. Similar cavities have been designed for use in B-factories, where this bunch rotation would allow head-on collisions with beams at a finite crossing angle [AKA92], and also as deflecting cavities for separating beams [BEL01].

Using the second harmonic of the accelerating rf frequency, the required peak transverse deflecting beam voltage is approximately $9 \mathrm{MV}$, and a multi-cell dipole mode structure is envisaged to provide this impedance. An asymmetric cavity shape enhances the desired dipole mode impedance in the vertical direction. The transverse deflection arises primarily from the interaction with the magnetic field in the cavity mode, however there is a significant contribution from the deflecting electric field in the associated TE-like mode in the beampipes adjoining the cavity.

Phase and amplitude stability of the cavity voltage is important to maintain synchronicity of the emitted x-rays with the experiment pump lasers, and for stability of the x-ray pulse length (Section 10). Future studies are aimed at developing rf systems synchronized to the experiment laser trigger, providing $\mathrm{x}$ ray to optical pulse jitter of approximately $10 \mathrm{fs}$. 


\section{Cryogenics Systems}

As described earlier, the pulse repetition rate of $10 \mathrm{kHz}$ requires that the superconducting structures run in $\mathrm{CW}$ mode. The power deposited in the walls of the cavities results from the superconducting surface resistance at the rf frequency. Additional losses resulting from higher order mode (HOM) power dissipated in the cavities are negligible for our low beam current. In addition to these dynamic rf losses, the cryogenic system must remove persistent or static losses due to conduction from surroundings outside the cold mass. These static losses include heat transferred through supports, input couplers, and HOM absorbers. Static losses for the TESLA cryomodules operating at $2^{\circ} \mathrm{K}$ are $0.1 \mathrm{Wm}^{-1}$ or $1.2 \mathrm{~W}$ for each (12-cavity) cryomodule [TES01], small compared to our dynamic losses of $336 \mathrm{~W}$ per main linac cryomodule and $184 \mathrm{~W}$ in the injector linac cryomodule.

The BCS theory describes the superconducting surface impedance, and for niobium the rf losses at frequencies below $10^{12} \mathrm{~Hz}$ and temperatures below half the transition temperature $T_{c}\left(\right.$ for $N b, T_{c}=9.2 \mathrm{~K}$ ) may be expressed by:

$$
R_{B C S}(\Omega)=2 \times 10^{-4} \frac{1}{T}\left(\frac{f}{1.5}\right)^{2} e^{-\frac{17.67}{T}}
$$

where $T$ is the operating temperature in Kelvin and $f$ is the frequency in GHz [PAD98]. Note that the exponential factor dominates the temperature dependence, and losses increase by a factor of 41 when operating at $4 \mathrm{~K}$ compared to operating at $2 \mathrm{~K}$. Consequently, there appears to be no advantage to operating at higher temperature.

The power deposited in the cryogenic system is the sum of static and dynamic loads described above. The wall-plug power required for the cryogenic system results from the deposited power divided by the Carnot efficiency and the technical efficiency of the system. Typical technical efficiency is $20 \%$, and the Carnot efficiency is given by:

$$
\eta_{\text {Carnot }}=\frac{T}{300-T}
$$

and the wall-plug power requirement is:

$$
P_{\text {cryogenic operations }}=\frac{P_{\text {cryogenic load }}}{\eta_{\text {Carnot }} \eta_{\text {technical }}}
$$

The cryogenic power load at $2 \mathrm{~K}$ is $1.53 \mathrm{~kW}$. With a $50 \%$ margin on this cryogenic load to allow flexibility for operations at higher cavity voltage, and to allow overhead for transfer line losses etc, the cryogenic system is then specified for a $2 \mathrm{~K}$ load of $2.3 \mathrm{~kW}$, and the a.c. power requirement is $1.7 \mathrm{MW}$. Table 7 summarizes the cryogenic system requirements.

TESLA cryomodules are designed for much lower $2 \mathrm{~K}$ loads of only $9 \mathrm{~W}$, and further design study is needed to determine system requirements and solutions for significantly higher power deposition per cryomodule. 
Table 7. Cryogenic system requirements

\begin{tabular}{|l|l|}
\hline Cryogenic dynamic load & $1.53 \mathrm{~kW}$ \\
\hline Cryogenic static load & $6 \mathrm{~W}$ \\
\hline Cryogenic temperature & $2 \mathrm{~K}$ \\
\hline Carnot efficiency & $0.67 \%$ \\
\hline Technical efficiency & $20 \%$ \\
\hline Estimated system load @ 2 K & $1.5 \mathrm{~kW}$ \\
\hline Specified system load capability @ 2 K & $2.3 \mathrm{~kW}$ \\
\hline Total a.c. power for cryogenic systems & $1.7 \mathrm{MW}$ \\
\hline
\end{tabular}

\section{Collective Effects}

The electromagnetic fields associated with an electron passing through a vacuum chamber interact with structures, leaving behind an electromagnetic field called the wake field. The primary effect of concern is the transverse deflection of trailing electrons within a bunch caused by the wake fields generated by leading electrons in the bunch, potentially creating a condition for a runaway instability. An additional effect is caused by wakefields persisting from preceding passes of the bunch through the linac - fortunately, the threshold beam current for such an instability is an average beam current far above the $80 \mu \mathrm{A}$ (eight passes of the linac during the acceleration/deceleration cycle) considered for nominal operation.

Under certain conditions the more deleterious single-pass effect may cause a significant growth of the electron beam emittance. This is a result of the transverse displacement of electrons toward the tail of the bunch. This effect may be neutralized by appropriate measures to control the phase of the interaction of the bunch with it's wakefield on each pass through the linac.

The transverse displacement $y(s, z)$ of trailing electrons due to the interaction with the wake field produced by the leading electrons may be written in the form [CHA80]:

$$
\frac{d}{d s}\left[\gamma(s) \frac{d y}{d s}\right]+k^{2}(s) \gamma(s) y(s, z)=r_{0} \int_{z}^{\infty} \rho\left(z^{\prime}\right) W_{\perp}\left(z^{\prime}-z\right)\left[y\left(s, z^{\prime}\right)-u(s)\right] d z^{\prime}
$$

where $\gamma$ is the relativistic factor, $k$ is the focusing strength (since there are no magnetic focusing elements within the recirculating linac, $k$ is only due to weak focusing by the rf fields), $u(s)$ is the alignment error of the rf cavities, $\rho$ is the electron bunch density, $s$ is the longitudinal position along the linac, $z$ is the internal bunch coordinate, $r_{0}$ is the classical electron radius, and $W_{\perp}$ is the transverse wake function per unit length. $W_{\perp}(\mathrm{z})$ for the linac cavity is shown in Figure 13 [MOS93]. 


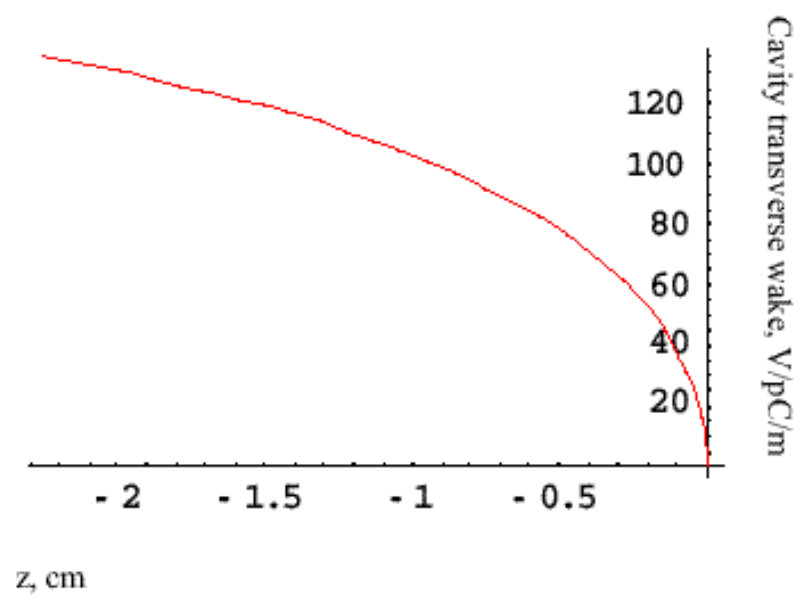

Figure 13. Transverse wake field of the TESLA linac cavity.

For a reasonably weak wakefield, where the wakefield at the center of the bunch $W_{\perp 0}$ is:

$$
W_{\perp 0} \leq \frac{15 \gamma_{0}}{N_{e} r_{0} L^{2}}
$$

and $L$ is the linac length, $N_{e}$ is the number of electrons in the bunch, and $\gamma_{0}$ is the electron beam energy in the middle of the linac, Eq.13 can be solved analytically and one can show that emittance growth can be mitigated. By generating a $(2 n+1) \pi$ phase advance of betatron oscillations between the first and the second passage of the linac and $2 n \pi$ phase advance for all following passages, where $n$ is an integer, the electron displacements introduced in the first pass are partially compensated by the subsequent passes. Additionally, the electron beam entering the linac on the first pass may be off-set in order to minimize the wake field resulting from the misalignments of linac cavities and cryomodules. For typical misalignment errors of 0.5 $\mathrm{mm}$ for individual cavities and $0.25 \mathrm{~mm}$ for cryomodules, the required orbit off-set for an electron bunch entering the recirculating linac is less than $0.1 \mathrm{~mm}$. Simulation results of application of these techniques are shown in Figure 14. Figure 14(a) shows the electron displacements $y / \sigma_{y}$ along the bunch with charge density $\rho(z)$, at the end of the fourth pass through the linac as a result of the rf structure misalignment errors. Figure 14(b) shows the displacement within a bunch after correction of the initial orbit off-set and selection of betatron phase advance discussed above. In the latter case, the resulting projected emittance growth is only a few percent.

The bunch lengths and beampipe apertures under current consideration result in a regime in which the coherent synchrotron radiation impedance may be significant, and further studies of such effects are in progress. A smaller beampipe aperture may be beneficial in reducing effects from coherent synchrotron radiation, but the minimum aperture may be limited by transverse wakefields arising from the resistive wall impedance.

Bunch length is easily adapted as required at different stages of acceleration and deceleration to provide flexibility with consideration to collective effects. Longitudinal bunch charge density may also be adjusted through variations in rf photocathode and incident laser beam parameters, should this prove useful in overcoming collective effects and improving machine performance. 

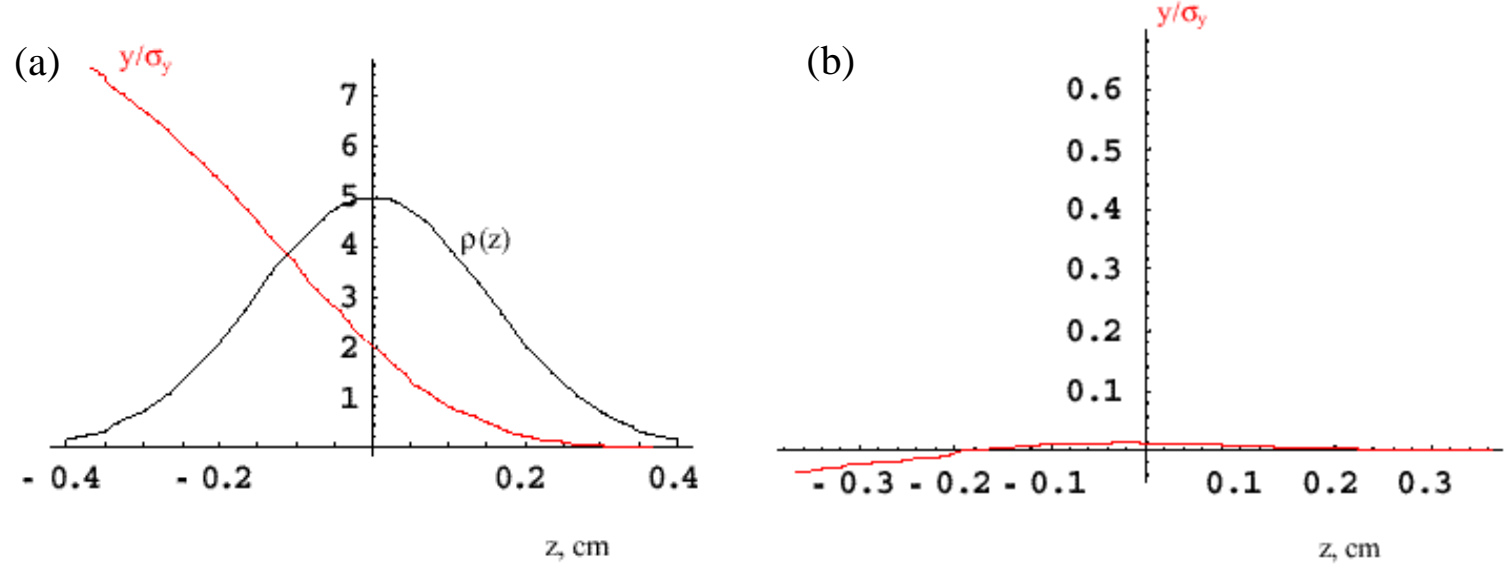

Figure 14. Normalized transverse displacement $y / \sigma_{z}$ along a bunch a) (left) with no corrective measures, b) (right) with initial transverse offset and betatron phase adjustment.

\section{Photon Production}

For a $2.5 \mathrm{GeV}$ electron beam of charge $1 \mathrm{nC}$ and repetition rate $10 \mathrm{kHz}$, the average photon flux from insertion devices and bend magnets is shown in Figures 15 and 16. In Figure 15, the photon flux as a function of photon energy is shown for an undulator of period $1.5 \mathrm{~cm}$, length $2 \mathrm{~m}$, undulator parameter $\mathrm{K}=2.1$ (peak magnetic field of $1.5 \mathrm{~T}$ ). A similar undulator is described in [ROS00]. Photon flux per (mrad) ${ }^{2}$ of bend magnet radiation for a $2 \mathrm{~T}$ bend magnet is shown in Figure 16.

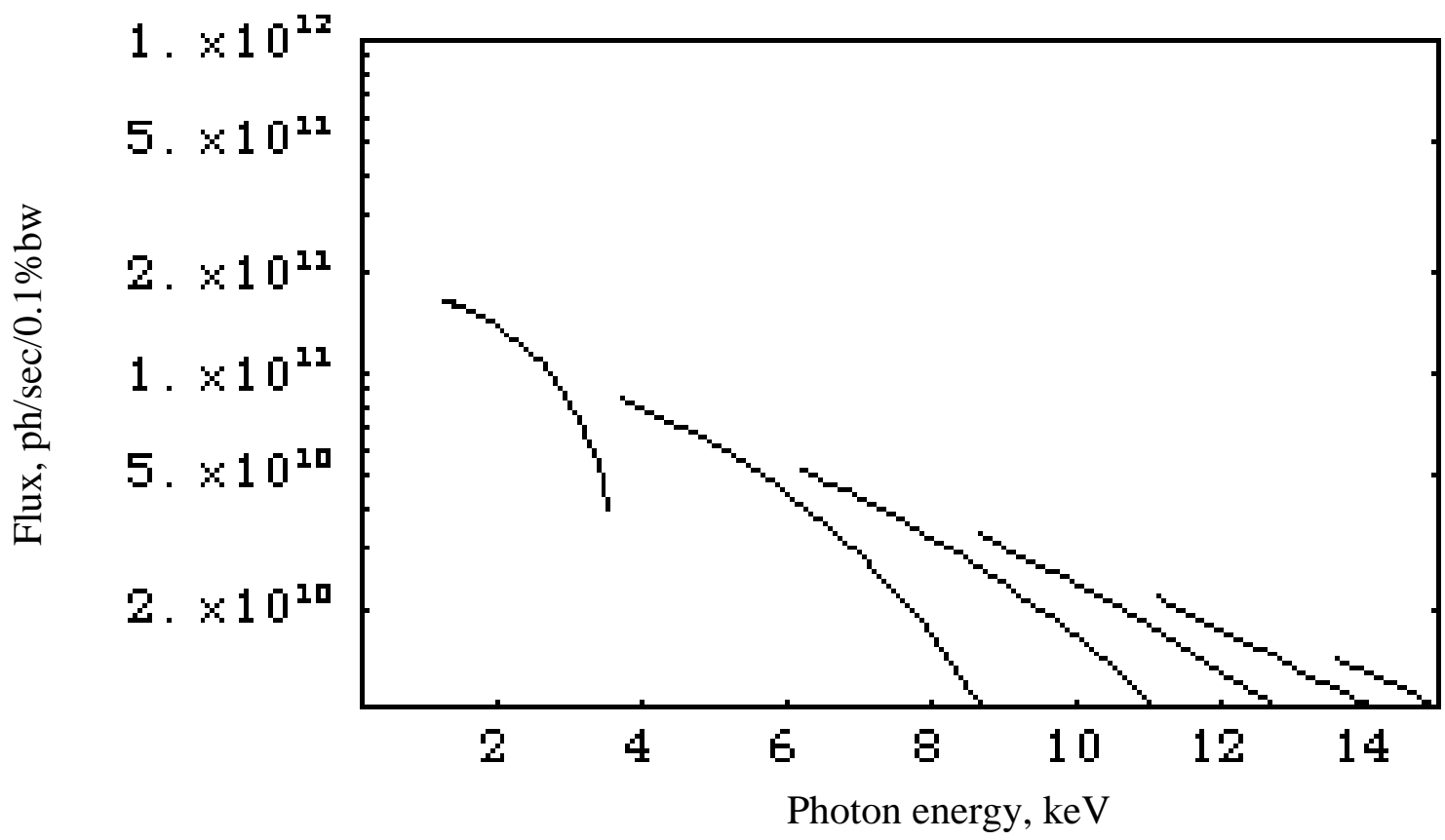

Figure 15. Undulator photon flux as a function of photon energy for the fundamental and lower odd harmonics. 


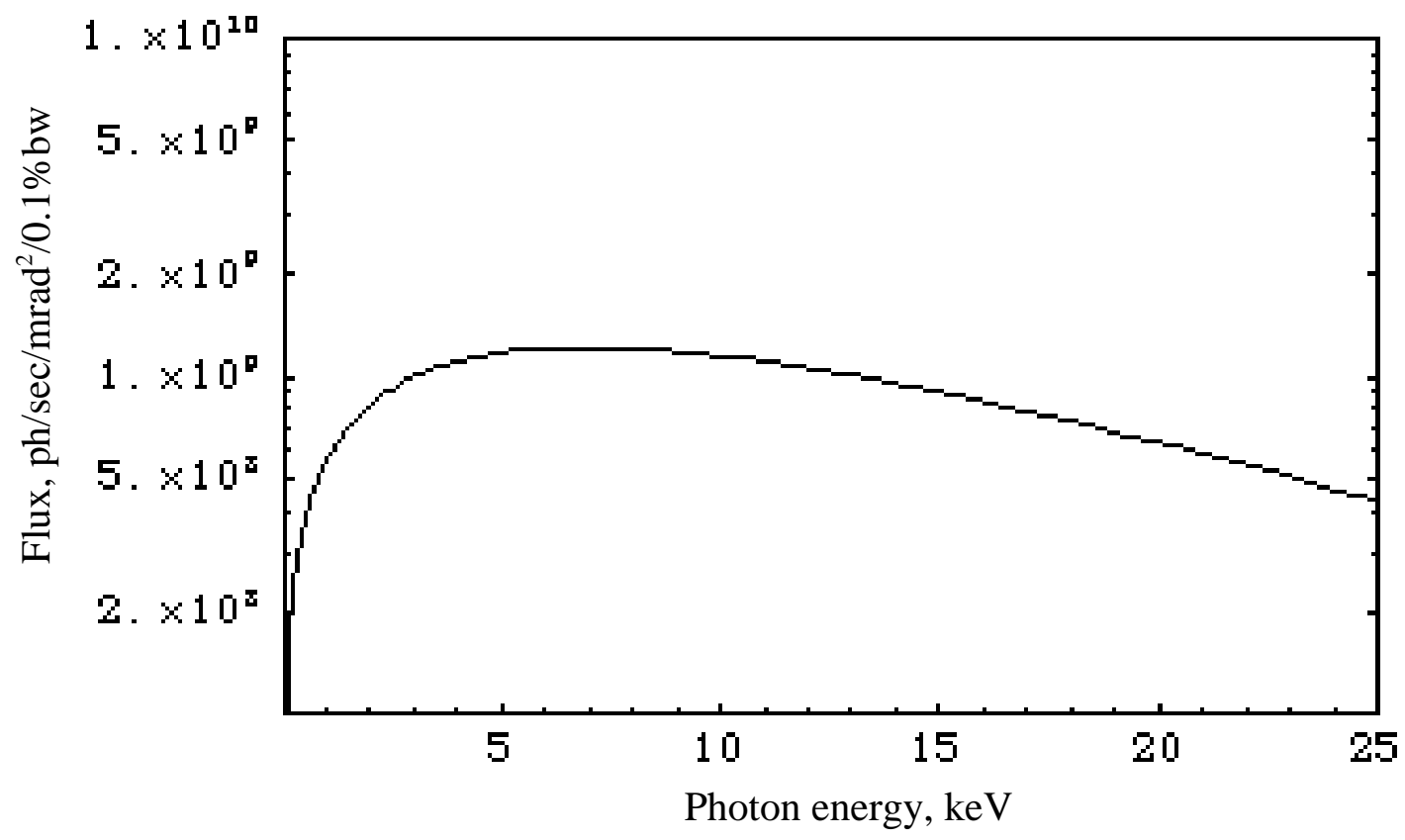

Figure 16. Photon flux as a function of photon energy for a $2 \mathrm{~T}$ field bend magnet.

\section{Synchronization of Laser and Femtosecond X-ray Pulses}

Most experiments utilizing the femtosecond x-ray pulse facility are expected to involve the use of femtosecond pump pulses from optical lasers. Synchronization between optical and x-ray pulses is required to be within a few tens of femtosecond in order to accurately map the ultra-fast dynamics initiated by the optical pulses. This requirement may be compromised by timing jitter between the electron and laser pulses.

Lasers systems have been locked to a common oscillator with better than ten femtosecond timing accuracy, as has been demonstrated in practice [MIU00]. Figure 17 demonstrates a standard means of locking the laser oscillator to the rf master oscillator (clock) of the accelerator. The rf frequency of the linac is $1.3 \mathrm{GHz}$, corresponding approximately to a 19th harmonic of a commercially available Ti: Sapphire laser oscillator. This harmonic is generated by a fast avalanche photodiode followed by a high Q filter with central frequency equal to that of the rf master oscillator. The signal thus generated from the laser and the signal from the rf master oscillator are applied to a mixer, the output of which is applied to the driver of a piezo-ceramic mirror mount to control the frequency of the laser oscillator. Using such a system, timing jitter between the amplified laser pulse and the RF phase has been demonstrated to be less than $\sim 1 \mathrm{ps}$ [ROD89]. 


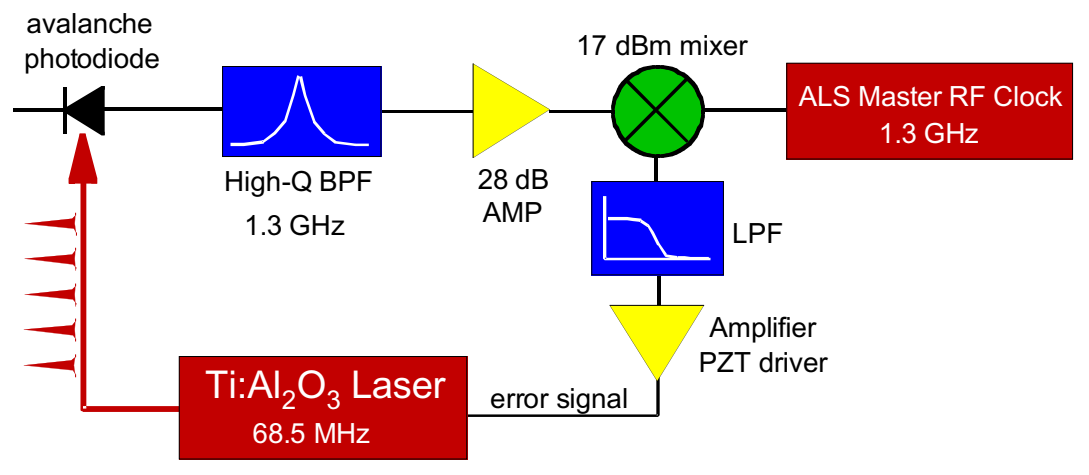

Figure 17. Schematic of a technique to synchronize the laser oscillator to the rf master oscillator (clock) of the linac.

Similarly, one may apply a signal derived from the laser oscillator to drive a klystron used for the rf deflection cavities. The resulting electron bunch deflections produced by the cavity will carry an imprint of the laser timing, and an aperture-limited system will produce a compressed x-ray pulse synchronized with the laser pulse independently of the electron pulse jitter. This is shown schematically in Figure 18. The magnitude of the remaining jitter between driver laser and phase of the rf field in the deflection cavity is presently under investigation.
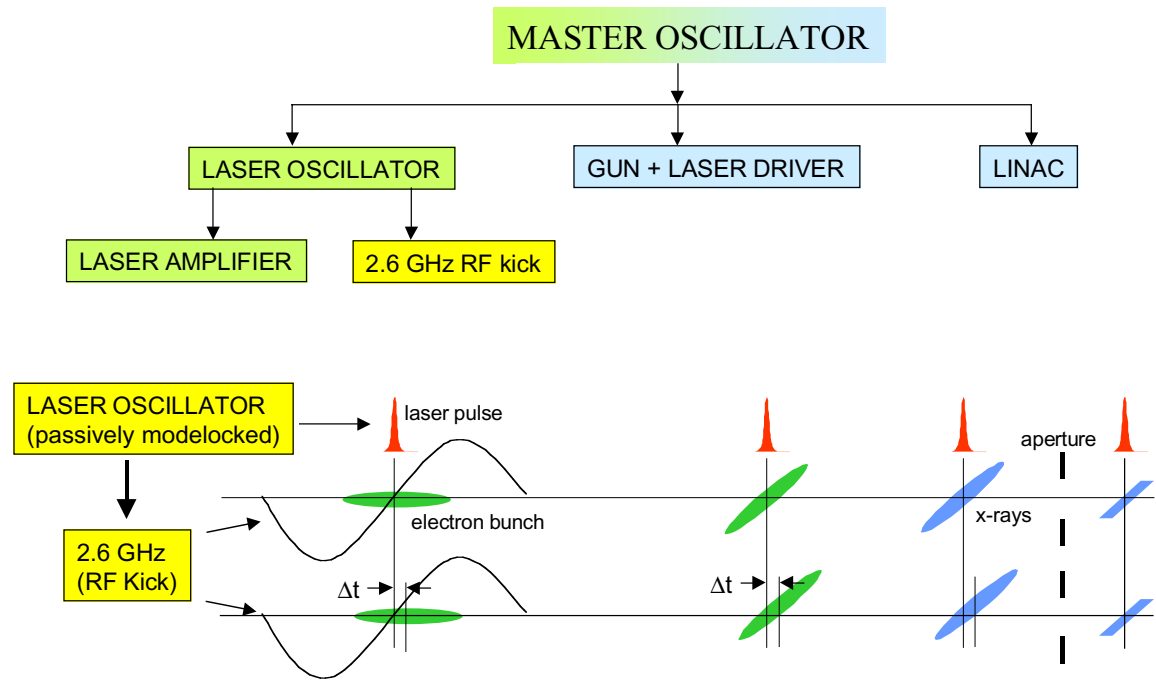

Figure 17. Schematic of synchronization of the laser and x-ray pulses. 


\section{Summary and Future Directions}

We have presented a concept for an accelerator based user facility to perform femtosecond x-ray science. The accelerator design is based on existing but state-of-the-art technologies which require some further development. Key areas to be studied are: high-quality rf photocathode guns, generation of beams with a large $\mathrm{x} / \mathrm{y}$ emittance ratio, control of beam emittance throughout the accelerator, efficient powering of the superconducting rf structures, exploration of cryogenics systems requirements, design of deflecting cavities, $\mathrm{x}$-ray beamline optics for pulse compression, and timing of the beam and x-ray pulse with respect to experimental systems. We propose to collaborate with various institutions and with industry in pursuit of solutions to technical problems.

Development of high-quality rf guns has proceeded at several institutions and we incorporate their successes into our design. We will proceed with designs based on a re-entrant nose-cone geometry, including beam dynamics calculations to ensure beam quality from such novel rf gun designs.

One important collaboration already entered into is in the production of a high-quality beam with large transverse aspect ratio in the injector. To further our understanding and pursue limitations of this technique, we are actively involved in experimental work at the Fermilab A0 photo-injector facility, in addition to performing simulation studies. The successful demonstration at the A0 facility of flat beams with emittance ratio 50:1 is highly encouraging and we have joined the experiment collaboration to help further work in this regard. Areas of specific interest include, for example, the maximum emittance ratio, and minimum emittance, as a function of bunch charge, energy spread, and bunch length.

Emittance control and understanding of collective effects is critical, and we are addressing key aspects of accelerator physics involved in beam break-up, coherent synchrotron radiation, and the influence of resistive wall wakefields. The electron bunch length at different stages of acceleration and deceleration is expected to be further refined after more comprehensive simulations. With several linac passes and many arcs we believe we have sufficient flexibility to optimally control the bunch length with consideration to collective effects. In addition, we may gain by manipulation of the longitudinal charge density profile of the electron beam emitted from the rf photocathode.

Superconducting rf structures are based on TESLA designs, and we plan to collaborate with industry and superconducting rf accelerator facilities to gain expertise in this area, and also derive cryogenics system limitations and design requirements. Since only a small fraction of the incident power is deposited in a cavity, we are investigating means to improve the efficiency of the system. Reducing the cavity bandwidth is advantageous and we will pursue technologies allowing feedback control of cavity resonant frequency at reduced bandwidths. Details of rf power distribution and control, and availability of rf power amplifiers, will be addressed. TESLA cryomodules are designed for $2 \mathrm{~K}$ loads of only $9 \mathrm{~W}$, and further design study is needed to determine system requirements and solutions for significantly higher power deposition per cryomodule.

Requirements for instrumentation and diagnostics will be addressed, including development of beam position monitors, transverse beam profile monitors, longitudinal profile monitors, and bunch charge monitors. Instrumentation will be necessary for each recirculating arc, and different techniques are likely to be employed to cover the large energy range. 
Beam timing techniques will be explored in collaboration with existing groups working with lasers locked to associated rf systems. Exploration of such techniques applied to non-linear elements such as klystrons will be pursued.

Close collaboration with the fs scientific community will be nurtured to ensure development of a facility geared to the needs of the science.

\section{References}

[ZHO99] A. Zholents, P. Heimann, M. Zolotorev, J. Byrd, "Generation of subpicosecond x-ray pulses using RF orbit deflection”, NIM A 425 (1999)385-389.

[BRI99] R. Brinkmann, Y. Derbenev, K. Flottmann, "A Flat-Beam Electron Source for Linear Colliders", DESY Report No. TESLA 99-09, 1999.

[BRI01] R. Brinkmann, Y. Derbenev, K. Flottmann, "A low emittance, flat-beam electron source for linear colliders", Phys. Rev. STAB, Vol. 4, 053501 (2001).

[MAT93] T. Matsushita and H. Hashizume, Handbook of Synchrotron Radiation V.1, ed. E.E. Koch, p.261, North-Holland, Amsterdam (1993).

[SMI94] R.K. Smither, P.B. Fernandez, Nuclear Instrum. and Methods A 347, 313 (1994).

[DER98] Y. Derbenev, "Adapting Optics for High Energy Electron Cooling", University of Michigan Report No. UM-HE-98-04, 1998.

[EDW00] D. Edwards et al, "The Flat Beam Experiment at the FNAL Photoinjector", Proc. XXth International Linac Conference, Monterey, 2000 (to be published).

[PER01] Proceedings of the PERL Photocathode Gun Workshop, Brookhaven National Laboratory, January 20-22, 2001. http://nslsweb.nsls.bnl.gov/nsls/org/PERL/GunWorkshop.htm

[TES01] TESLA Technical Design Report, DESY 2001-011, March 2001.

[AKA92] K. Akai, J. Kirchgessner, D. Moffat, H. Padamsee, J. Sears, T. Stowe, M. Tigner, "Crab Cavity for the B-Factories", Proceedings of B Factories The State of the Art in Accelerators, Detectors and Physics, Stanford Linear Accelerator Center, April 1992, SLAC-400, CONF-9204126.

[BEL01] L. Bellantoni, H.T., Edwards, M. McAshan, R. Wanzenberg, "Design and measurements of a deflecting mode cavity for an rf separator", Proc. 2001 Particle Accelerator Conference, Chicago, 2001; http://pacwebserver.fnal.gov/papers/Monday/PM_Poster/MPPH129.pdf

[PAD98] H. Padamse, J. Knobloch, T. Hays, "RF Superconductivity for Accelerators", John Wiley \& Sons Inc, ISBN 0-471-15432-6, 1998.

[CHA80] A. Chao, B. Richter, C.-Y. Yao, " Beam Emittance Growth Caused by Transverse Deflecting Fields in a Linear Accelerator ", SLAC-PUB-2498 (1980). 
[MOS93] A. Mosnier, " Longitudinal and Transverse Wakes for the TESLA Cavity", TESLA 9311 (1993).

[ROS00] R. Rossmanith, H.O. Moser, "Design study of a superconductive in-vacuo undulator", Proc. of European Particle Accelerator Conference, EPAC 2000, Vienna, 2000.

[MIU00] T. Miura, K. Kobayashi, K. Takasago, ZG Zhang, ZG "Timing jitter in a kilohertz regenerative amplifier of a femtosecond-pulse Ti : Al2O3 laser", Optics Letters, 2000 Dec 15, V25 N24:1795-1797.

[ROD89] M.J. Rodwell, D.M. Bloom, K.J. Weingarten, " Subpicosecond laser timing stabilization", IEEE J. Quantum Elctron. 25 (No. 4), pp.817-827 (1989). 